\title{
Multisection in the Stochastic Block Model using Semidefinite Programming
}

\author{
Naman Agarwal, Afonso S. Bandeirał Konstantinos Koiliaris; Alexandra Kolla ${ }^{\ddagger}$
}

July 10, 2015

\begin{abstract}
We consider the problem of identifying underlying community-like structures in graphs. Towards this end we study the Stochastic Block Model (SBM) on $k$-clusters: a random model on $n=k m$ vertices, partitioned in $k$ equal sized clusters, with edges sampled independently across clusters with probability $q$ and within clusters with probability $p, p>q$. The goal is to recover the initial "hidden" partition of $[n]$. We study semidefinite programming (SDP) based algorithms in this context. In the regime $p=\frac{\alpha \log (m)}{m}$ and $q=\frac{\beta \log (m)}{m}$ we show that a certain natural SDP based algorithm solves the problem of exact recovery in the $k$-community SBM, with high probability, whenever $\sqrt{\alpha}-\sqrt{\beta}>\sqrt{1}$, as long as $k=o(\log n)$. This threshold is known to be the information theoretically optimal. We also study the case when $k=\theta(\log (n))$. In this case however we achieve recovery guarantees that no longer match the optimal condition $\sqrt{\alpha}-\sqrt{\beta}>\sqrt{1}$, thus leaving achieving optimality for this range an open question.
\end{abstract}

Keywords: graph partitioning, random models, stochastic block model, semidefinite programming, dual certificate

\footnotetext{
*namana@cs.princeton.edu, Computer Science, Princeton University

†bandeira@mit.edu, Department of Mathematics, Massachusetts Institute of Technology (most of the work presented in this paper was conducted while this author was at Princeton University). ASB acknowledges support from AFOSR Grant No. FA9550-12-1-0317

${ }^{\ddagger}$ koiliar2@illinois.edu, Computer Science, University of Illinois, Urbana - Champaign

$\S$ akolla@illinois.edu, Computer Science, University of Illinois, Urbana - Champaign
} 


\section{Introduction}

Identifying underlying structure in graphs is a primitive question for scientists: can existing communities be located in a large graph? Is it possible to partition the vertices of a graph into strongly connected clusters? Several of these questions have been shown to be hard to answer, even approximately, so instead of looking for worst-case guarantees attention has shifted towards average-case analyses. In order to study such questions, the usual approach is to consider a random [McS01] or a semi-random [FK01, MMV14] generative model of graphs, and use it as a benchmark to test existing algorithms or to develop new ones. With respect to identifying underlying community structure, the Stochastic Block Model (SBM) (or planted partition model) has, in recent times, been one of the most popular choices. Its growing popularity is largely due to the fact that its structure is simple to describe, but at the same time it has interesting and involved phase transition properties which have only recently been discovered ([DKMZ11, MNS12, MNS13, ABH14, CX14, MNS14b, HWX14, HWX15, AS15, Ban15]).

In this paper we consider the SBM on $k$-communities defined as follows. Let $n$ be a multiple of $m, V=[n]$ be the set of vertices and $P=\left\{P_{i}\right\}$ be a partition of them into $k$ equal sized clusters each of size $m=\frac{n}{k}$. Construct a random graph $G$ on $V$ by adding an edge for any two vertices in the same cluster independently with probability $p$ and any two vertices across distinct clusters independently with probability $q$ where $p>q$. We will write $G \sim \mathcal{G}_{p, q, k}$ to denote that a graph $G$ is generated from the above model. Given such a $G$ the goal is to recover (with high probability) the initial hidden partition $P$.

The SBM can be seen as an extension of the Erdős-Rényi random graph model [ER59] with the additional property of possessing a non-trivial underlying community structure (something which the Erdős-Rényi model lacks). This richer structure not only makes this model interesting to study theoretically, but also renders it closer to real world inputs, which tend to have a community structure. It is also worth noting that, as pointed out in [CX14], a slight generalization of the SBM encompasses several classical planted random graph problems including planted clique [AKS98], [McS01], planted coloring [AK97], planted dense subgraph [AV13] and planted partition [Bop87, CK01, FK01].

There are two natural problems that arise in context of the SBM: exact recovery, where the aim is to recover the hidden partition completely; and detection, where the aim is to recover the partition better than what a random guess would achieve. In this paper we focus on exact recovery. Note that exact recovery necessarily requires the hidden clusters to be connected (since otherwise there would be no way to match the partitions in one component to another component) and it is easy to see that the threshold for connectivity occurs when $p=\Omega(\log (m) / m)$. Therefore the right scale for the threshold behavior of the parameters $p, q$ is $\Theta(\log (m) / m)$, which is what we consider in this paper.

In the case of two communities $(k=2)$ Abbe et al. [ABH14] recently established a sharp phase transition phenomenon from information-theoretic impossibility to computational feasibility of exact recovery. However, the existence of such a phenomenon in the case of $k>2$ was left open until solved, for $k=O(1)$, in independent parallel research [AS15, HWX15]. In this paper we resolve the above showing the existence of a sharp phase transition for $k=o(\log (n))$.

More precisely, in this work, we study a Semidefinite Programming (SDP) based algorithm that, for $k=o(\log (n))$, recovers, for an optimal range of parameters, exactly the planted $k$ partition of $G \sim \mathcal{G}_{p, q, k}$ with high probability. The range of the parameters $p, q$ is optimal in the following sense: it can be shown that this parameter range exhibits a sharp phase transition from 
information-theoretic impossibility to computational feasibility through the SDP algorithm studied in this paper. An interesting aspect of our result is that, for $k=o(\log (n))$, the threshold is the same as for $k=2$. This means that, even if an oracle reveals all of the cluster memberships except for two, the problem has essentially the same difficulty. We also consider the case when $k=\Theta(\log (n))$. Unfortunately, in this regime we can no longer guarantee exact recovery up to the proposed information theoretic threshold. Similar behavior was observed and reported by Chen et al. [CX14] and in our work we observe that the divergence between our information theoretic lower bound and our computational upper bound sets in at $k=\Theta(\log (n))$. This is formally summarized in the following theorems.

Theorem 1.1. Given a graph $G \sim \mathcal{G}_{p, q, k}$ with $k=O(\log (m))$ hidden clusters each of size $m$ and $p=\frac{\alpha \log (m)}{m}$ and $q=\frac{\beta \log (m)}{m}$, where $\alpha>\beta>0$ are fixed constants, the semidefinite program (4), with probability $1-n^{-\Omega(1)}$, recovers the clusters when:

- for $k=o(\log n)$, as long as

$$
\sqrt{\alpha}-\sqrt{\beta}>1
$$

- for $k=(\gamma+o(1)) \log (n)$ for a fixed $\gamma$, as long as

$$
\sqrt{\alpha}-\sqrt{\beta}>\sqrt{1+c \sqrt{\beta \gamma}\left(1+\log \left(\sqrt{\frac{\alpha}{\beta}}\right)\right)},
$$

where $c$ is a universal constant.

We complement the above theorem by showing the following lower bound which is a straightforward extension of the lower bound for $k=2$ from [ABH14].

Theorem 1.2. Given a graph $G \sim \mathcal{G}_{p, q, k}$ with $k$ hidden clusters each of size $m$ where $k$ is $o\left(m^{-\lambda}\right)$ for any fixed $\lambda>0$, if $p=\frac{\alpha \log (m)}{m}$ and $q=\frac{\beta \log (m)}{m}$, where $\alpha>\beta>0$ are fixed constants, then it is information theoretically impossible to recover the clusters exactly with high probability if

$$
\sqrt{\alpha}-\sqrt{\beta}<1 .
$$

Note that Theorem 1.2 establishes a sharp phase transition between computational feasibility and information theoretic impossibility when $k=o(\log (n))$. At $k \sim \log (n)$ we see that our lower and upper bounds diverge. We leave as an open problem to determine whether such divergence is necessary or a shortcoming of the SDP approach.

At the heart of our argument is the following theorem which establishes a sufficient condition for exact recovery with high probability.

Theorem 1.3. Let $G \sim \mathcal{G}_{p, q, k}$, with probability $1-n^{-\Omega(1)}$ over the choice of $G$, if the following condition is satisfied, the semidefinite program (4) recovers the hidden partition:

$$
\min _{i} \Delta(i) \geq \hat{c}\left(\sqrt{p n / k+q n}+q \sqrt{\frac{n}{k} \log (n)}+\sqrt{\log (n)}+\log (k)\right),
$$

where $\hat{c}$ is a universal constant and $\Delta(i)$ is defined as the difference between the number of neighbors a vertex $i$ has in its own cluster and the maximum number of neighbors it has in any other cluster (with respect to the hidden partition). In other words, with probability $1-n^{-\Omega(1)}$, (1) implies exact recovery. 
We are able to give sharp guarantees for the semidefinite programming algorithm based essentially on the behavior of inner and outer degrees of the vertices. This is achieved by constructing a candidate dual certificate and using bounds on the spectral norm of random matrices to show that the constructed candidate is indeed a valid one. The problem is then reduced to the easier task of understanding the typical values of such degrees. Remarkably, the conditions required for these quantities are very similar to the ones required for the problem to be information-theoretically solvable (which essentially correspond to each node having larger in-degree than out-degree). This helps explain the optimality of our algorithm. The approach of reducing the validity of a dual certificate to conditions on an interpretable quantity appeared in [Ban15] for a considerably simpler class of problems where the dual certificate construction is straightforward (which includes the stochastic block model for $k=2$ but not $k>2$ ). In contrast, in the current setting, the dual certificate construction is complex, rendering a different, and considerably more involved analysis. Moreover, the estimates we need (both of spectral norms and of inner and outer degrees) do not fall under the class of the ones studied in [Ban15].

We also show that our algorithm recovers the planted partitions exactly also in the presence of a monotone adversary, a semi-random model defined in [FK01].

\subsection{Related Previous and Parallel Work}

Graph partitioning problem has been studied over the years with various different objectives and guarantees. There has been significant recent literature concentration around the bipartiton (bisection) and the general $k$-partition problems (multisection) in random and semi-random models ([DKMZ11], [MNS12], [MNS13], [YP14], [MNS14a], [Mas14], [ABH14], [CX14], [MNS14b], [Vu14], [CRV15]).

Some of the first results on partitioning random graphs were due to Bui et al. [BCLS84] who presented algorithms for finding bipartitions in dense graphs. Boppana [Bop87] showed a spectral algorithm that for a large range of parameters recovers a planted bipartition in a graph. Feige and Kilian [FK01] present an SDP based algorithm to solve the problem of planted bipartition (along with the problems of finding Independent Sets and Graph Coloring). Independently, McSherry [McS01] gave a spectral algorithm that solved the problems of Multisection, Clique and Graph Coloring.

More recently, a spate of results have established very interesting phase transition phenomena for SBMs, both for the case of detection and exact recovery. For the case of detection, where the aim is to recover partitions better than a random guess asymptotically, recent works of [MNS12, MNS13, Mas14] established a striking sharp phase transition from information theoretic impossibility to computational feasibility for the case of $k=2$. For the case of exact recovery Abbe et al. [ABH14], and independently [MNS14b], established the existence of a similar phase transition phenomenon albeit at a different parameter range. More recently the same phenomenon was shown to exist for a semidefinite programming relaxation, for $k=2$ in [HWX14, Ban15]. However, the works described above established phase transition for $k=2$ and the case for larger $k$ was left open. Our paper bridges the gap for larger $k$ upto $o(\log (n))$ for the case of exact recovery. To put our work into context, the corresponding case of establishing such behavior for the problem of detection remains open. In fact, it is conjectured in [DKMZ11, MNS12] that, for the detection problem, there exists a gap between the thresholds for computational feasibility and information theoretic impossibility for any $k$ number of communities greater than 4 . In this paper, we show that that is not case for the exact recovery problem. 
Chen et al. [CX14] also study the $k$-community SBM and provide convex programming based algorithms and information theoretic lower bounds for exact recovery. Their results are similar to ours in the sense that they also conjecture a separation between information theoretic impossibility and computation feasibility as $k$ grows. In comparison we focus strongly on the case of slightly superconstant $k(o(\log (n)))$ and mildly growing $k(\Omega(\log (n)))$ and show exact recovery to the optimal (even up to constants) threshold in the former case. Very recently in independent and parallel work, Abbe and Sandon [AS15] studied the problem of exact recovery for a fixed number of $(k>2)$ communities where the symmetry constraint (equality of cluster sizes and the probabilities of connection are same in different clusters) is removed. Our result, in contrast to theirs, is based on the integrality of a semidefinite relaxation, which has the added benefit of producing an explicit certificate for optimality (i.e. indeed when the solution is "integral" we know for sure that it is the optimal balanced $k$-partition). Abbe and Sandon [AS15] comment in their paper that their results can be extended for slightly superconstant $k$ but leave it as future work. In another parallel and independent work, Hajek et al. [HWX15] study semidefinite programming relaxations for exact recovery in SBMs and achieve similar results as ours. We remark that semidefinite program in consideration in [HWX15] is the same as the semidefinite program (4) considered by us (up to an additive/multiplicative shift) and both works achieve the same optimality guarantee for $k=O(1)$. They also consider the problem of SBM with 2 unequal sized clusters and the Binary Censored Block Model. In contrast we show that the guarantees extend to the case even $k$ is superconstant $o(\log (n))$ and provide sufficient guarantees for the case of $k=\theta(\log (n))$ pointing to a possible divergence between information theoretic possiblity and computational feasibility at $k=\log (n)$ which we leave as an open question.

\subsection{Preliminaries}

In this section we describe the notation and definitions which we use through the rest of the paper.

Notation. Throughout the rest of the paper we will be reserving capital letters such as $X$ for matrices and with $X[i, j]$ we will denote the corresponding entries. In particular, $J$ will be used to denote the all ones matrix and $I$ the identity matrix. Let $A \bullet B$ be the element wise inner product of two matrices, i.e. $A \bullet B=\operatorname{Trace}\left(A^{T} B\right)$. We note that the all the logarithms used in this paper are natural logarithms i.e. with the base $e$.

Let $G=(V, E)$ be a graph, $n$ the number of vertices and $A(G)$ its adjacency matrix. With $G \sim \mathcal{G}_{p, q, k}$ we denote a graph drawn from the stochastic block model distribution as described earlier with $k$ denoting the number of hidden clusters each of size $m$. We denote the underlying hidden partition with $\left\{P_{t}\right\}$. Let $P(i)$ be the function that maps vertex $i$ to the cluster containing $i$. To avoid confusion in the notation note that with $P_{t}$ we denote the $t^{\text {th }}$ cluster and $P(i)$ denotes the cluster containing the vertex $i$. We now describe the definitions of a few quantities which will be useful in further discussion of our results as well as their proofs. Define $\delta_{i \rightarrow P_{t}}$ to be the "degree" of vertex $i$ to cluster $t$. Formally

$$
\delta_{i \rightarrow P_{t}} \triangleq \sum_{j \in P_{t}} A(G)[i, j]
$$

Similarly for any two clusters $P_{t_{1}}, P_{t_{2}}$ define $\delta_{P_{t_{1}} \rightarrow P_{t_{2}}}$ as

$$
\delta_{P_{t_{1}} \rightarrow P_{t_{2}}} \triangleq \sum_{i \in P_{t_{1}}} \sum_{j \in P_{t_{2}}} A(G)[i, j]
$$


Define the "in degree" of a vertex $i$, denoted $\delta^{i n}(i)$, to be the number of edges of going from the vertex to its own cluster

$$
\delta^{i n}(i) \triangleq \delta_{i \rightarrow P(i)},
$$

also define $\delta_{\max }^{\text {out }}(i)$ to be the maximum "out degree" of a vertex $i$ to any other cluster

$$
\delta_{\max }^{\text {out }}(i) \triangleq \max _{P_{t} \neq P(i)} \delta_{i \rightarrow P_{t}}
$$

Finally, define

$$
\Delta(i) \triangleq \delta^{\text {in }}(i)-\delta_{\max }^{\text {out }}(i),
$$

$\Delta(i)$ will be the crucial parameter in our threshold. Remember that $\Delta(i)$ for $A(G)$ is a random variable and let $\Delta \triangleq \mathbb{E}[\Delta(i)]$ be its expectation (same for all $i$ ).

Paper Organization. The rest of this paper is structured as follows. In Section 2 we discuss the two SDP relaxations we consider in the paper. We state sufficient conditions for exact recovery for both of them as Theorem 2.1 and Theorem 2.2 (the latter is a restatement of Theorem 1.3) and provide an intuitive explanation of why the condition (1) is sufficient for recovery upto the optimal threshold. We provide formal proofs of Theorems 1.1 and 1.2 in the Appendix in Sections A.4 and A.3 respectively. We provide the proof of Theorem 2.2 in Section 3. Further in Section 4 we show how our result can be extended to a semi random model with a monotone adversary. Lastly in the Appendix we collect the proofs of all the lemmas and theorems left unproven in the main sections.

\section{SDP relaxations and main results}

In this section we present two candidate SDPs which we use to recover the hidden partition. The first SDP is inspired from the Max-k-Cut SDP introduced by Frieze and Jerrum [FJ95] where we do not explicitly encode the fact that each cluster contains equal number of vertices. In the second SDP we encode the fact that each cluster has exactly $m$ vertices explicitly. We state our main theorems which provide sufficient conditions for exact recovery in both SDPs. Indeed the latter SDP, being stronger, is the one we use to prove our main theorem, Theorem 1.1. Before describing the SDPs lets first consider the Maximum Likelihood Estimator (MLE) of the hidden partition. It is easy to see that the MLE corresponds to the following problem which we refer to as the Multisection problem. Given a graph $G=(V, E)$ divide the set of vertices into $k$ clusters $\left\{P_{t}\right\}$ such that for all $t_{1}, t_{2},\left|P_{t_{1}}\right|=\left|P_{t_{2}}\right|$ and the number of edges $(u, v) \in E$ such that $u \in P_{t_{1}}$ and $v \in P_{t_{2}}$ are minimized. (This problem has been studied under the name of Min-Balanced-kpartition [KNS09]). In this section we consider two SDP relaxations for the Multisection problem. Since SDPs can be solved in polynomial time, the relaxations provide polynomial time algorithms to recover the hidden partitions.

A natural relaxation to consider for the problem of multisection in the Stochastic Block Model is the Min-k-cut SDP relaxation studied by Frieze and Jerrum [FJ95] (They actually study the Max-k-Cut problem but we can analogously study the min cut version too). The Min-k-cut SDP formulates the problem as an instance of Min-k-cut where one tries to separate the graph into $k$ partitions with the objective of minimizing the number of edges cut by the partition. Note that the $\mathrm{k}$-Cut version does not have any explicit constraints for ensuring balancedness. However studying Min-k-Cut through SDPs has a natural difficulty, the relaxation must explicitly contain a constraint 
that tells it to divide the graph into at least $k$ clusters. In the case of SBMs with the parameters $\alpha \frac{\log (n)}{n}$ and $\beta \frac{\log (n)}{n}$ one can try and overcome the above difficulty by making use of the fact that the generated graph is very sparse. Thus, instead of looking directly at the min-k-cut objective we can consider the following objective: minimizing the difference between the number of edges cut and the number of non-edges cut. Indeed for sparse graphs the second term in the difference is the dominant term and hence the SDP has an incentive to produce more clusters. Note that the above objective can also be thought of as doing Min-k-Cut on the signed adjacency matrix $2 A(G)-J$ (where $J$ is the all ones matrix). Following the above intuition we consider the following SDP (2) which is inspired from the Max-k-Cut formulation of Feige and Jerrum [FJ95]. In the Appendix Section A.2 we provide a reduction, to the k-Cut SDP we study in this paper, from a more general class of SDPs studied by Charikar et al. [CMM06] for Unique Games, and more recently by Bandeira et al. [BCS15] in a more general setting.

$$
\begin{array}{ll}
\max & (2 A(G)-J) \bullet Y \\
\text { s.t. } & Y_{i i}=1(\forall i) \\
& Y_{i j} \geq-\frac{1}{k-1}(\forall i, j) \\
& Y \succcurlyeq 0 .
\end{array}
$$

To see that the above SDP is a relaxation of the multisection problem note that for the hidden partition $\left\{P_{t}\right\}$ we can define a candidate solution $Y^{*}$ as follows. $Y_{i j}^{*}=1$ if $i, j$ belong to the same cluster and $-\frac{1}{k-1}$ if $i, j$ belong to different clusters. Note that although the objective does not directly minimize the number of edges cut, it is an additive/multiplicative shift of it. For the above $\mathrm{SDP}$ we prove the following theorem in the Appendix in Section A.7.1. Given $G \sim \mathcal{G}_{p, q, k}$, define

$$
\nu(i) \triangleq \delta_{i n}(i)-\max _{i, j}\left(\delta_{i \rightarrow P(j)}+\delta_{j \rightarrow P(i)}-\frac{\delta_{P(j) \rightarrow P(i)}}{n / k}\right)
$$

Theorem 2.1. Let $G \sim \mathcal{G}_{p, q, k}$, with $p=\alpha \frac{\log (m)}{m}$ and $q=\beta \frac{\log (m)}{m}$ where $\alpha, \beta$ are constant. Consider the SDP given by (2). With probability $1-n^{-\Omega(1)}$ over the choice of $G$, if the following condition is satisfied then the SDP recovers the hidden partition

$$
\min _{i} \nu(i) \geq \hat{c}(\sqrt{p n / k+q n}+\sqrt{\log (n)})
$$

where $\hat{c}$ is a universal constant.

In other words with probability $1-n^{-\Omega(1)}$, condition (3) implies exact recovery.

The proof of the above Theorem is included in the Appendix in Section A.7.1. We note the above condition is not an optimal one in terms of exact recovery and we discuss this issue next. It is quite possible that the above SDP recovers the planted multisection all the way down to the threshold however we have not been able to establish this and leave it as an open question. Indeed to prove our results we consider a stronger SDP with which we establish optimality. We have empirically tested the performance of both the SDPs and include the results in the Appendix in Section A.1. We now take a closer look at the above sufficient condition (3) and argue why the condition is not strong enough to achieve optimal results. It is not hard to see that

$$
\mathbb{E}[\nu(i)] \sim p \frac{n}{k}-q \frac{n}{k}-O\left(\sqrt{q \frac{n}{k} \log (n)}\right)
$$


Note that, in expectation, the maximization term in the definition of $\nu(i)$ has an extra $\log (n)$ term as the maximization runs through all $i, j$ pairs. For the condition (3) to hold with at least a constant probability, we expect that it needs to be the case that

$$
p \frac{n}{k}-q \frac{n}{k}-O\left(\sqrt{q \frac{n}{k} \log (n)}\right) \geq O\left(\sqrt{p \frac{n}{k}+q \frac{n}{k} k}+\sqrt{\log (n)}\right)
$$

Substituting the parameter range that we are interested $p=\frac{\alpha \log (m)}{m}$ and $q=\frac{\beta \log (m)}{m}$ we require that

$$
\alpha-\beta \geq O\left(\sqrt{\beta}+\sqrt{\frac{\beta k}{\log (n)}}\right)
$$

Indeed from the above expression it is clear that if $k<<\log (n)$ the first term above dominates and we cannot expect to get the tight results we hope for in Theorem 1.1. A closer look at the above calculation reveals that the major barrier towards achieving the optimal result is the additional $\log (n)$ factor due to the maximization over all $i, j$ in the definition of $\nu(i)$. For instance if one could replace the maximization term above with a term that takes the maximum per vertex over all clusters one would pick up only a $\log (k)$ term (as there are only $k$ clusters) and hopefully achieve optimality.

In context of the above discussion we suggest the following SDP in which we explicitly add a per-row constraint bounding the number of vertices belonging to the same cluster as the vertex in contention.

$$
\begin{array}{ll}
\max & A(G) \bullet Y \\
\text { s.t. } & \sum_{j} Y_{i j}+\sum_{j} Y_{j i}=2 n / k \quad(\forall i) \\
& Y_{i i}=1 \quad(\forall i) \\
& Y_{i j} \geq 0 \quad(\forall i, j) \\
& Y \succcurlyeq 0 .
\end{array}
$$

To see that the above SDP is a relaxation of the MLE discussed above note that for any partition $P=\left\{P_{i}\right\}$, we can associate a canonical $n \times n$ matrix $Y_{P}$ with it defined as

$$
Y_{P}[i, j]= \begin{cases}1 & \text { vertex } i \text { and } j \text { belong to the same cluster } \\ 0 & \text { otherwise }\end{cases}
$$

Note that $Y_{P}$ satisfies the SDP constraints and the SDP maximizes the number of edges within the cluster which is equivalent to minimizing the number of edges across the clusters. The second constraint above, since $Y$ is symmetric, says that the sum of the values along the row is $n / k$, which represents the number of vertices in a cluster. For the SDP above we show the following theorem which is a restatement of Theorem 1.3

Theorem 2.2. Let $G \sim \mathcal{G}_{p, q, k}$. With probability $1-n^{-\Omega(1)}$ over the choice of $G$, if the following condition is satisfied then the SDP defined by (4) recovers the hidden partition

$$
\min _{i} \Delta(i) \geq \hat{c}\left(\sqrt{p n / k+q n}+q \sqrt{\frac{n}{k} \log (n)}+\sqrt{\log (n)}+\log (k)\right),
$$

In other words with probability $1-n^{-\Omega(1)}$, condition (5) implies exact recovery. 
We remark that the above statement is indeed true for all values of $p, q$. For the specific range that we are interested in we show in Section A.4 how condition (5) leads to the optimal threshold. In the next section we provide an intuitive explanation of why this is so.

\subsection{Optimality of Theorem 2.2}

In this section we give an intuitive high level explanation for the optimality of the condition in (5) for $k<<\log (n)$ in Theorem 2.2. We prove it formally in the appendix. As stated earlier the regime we consider is the case when $p=\frac{\alpha \log (m)}{m}$ and $q=\frac{\beta \log (m)}{m}$, where $\alpha$ and $\beta$ are constants.

Note that for the MLE to succeed the values of $p$ and $q$ should be such that $\min _{i}\left\{\delta^{i n}(i)-\right.$ $\left.\delta_{\max }^{\text {out }}(i)\right\} \geq 0$ w.h.p., since otherwise one expects there to be many such vertices $i$ for which $\delta^{\text {in }}(i)-$ $\delta_{i \rightarrow P_{t}} \leq 0$ for some $t \neq P(i)$ and in particular a pair $t_{1}, t_{2}$ such that there exists $i \in P_{t_{1}}, j \in P_{t_{2}}$ such that $\delta^{i n}(i)-\delta_{i \rightarrow P_{t_{2}}} \leq 0$ as well as $\delta^{i n}(j)-\delta_{i \rightarrow P_{t_{1}}} \leq 0$. This would imply that we can exchange the pairs $i, j$ and get a better partition than the planted partition and therefore that the MLE itself does not recover the hidden partition.

Recall that $\Delta(i)=\delta^{\text {in }}-\delta_{\max }^{\text {out }}(i)$. We now show that the deviation in $\Delta(i)$ required by Theorem 2.2 is $o(\mathbb{E}[\Delta(i)])$ and therefore informally one can expect, intuitively, that

$$
\mathbb{P}\left(\min _{i} \Delta(i) \geq 0\right) \sim \mathbb{P}\left(\min _{i} \Delta(i) \geq o(\mathbb{E}[\Delta(i)])\right)
$$

which implies that the SDP in Theorem 2.2 recovers the partition optimally. Indeed, the deviation required in Theorem 2.2 is $o(\mathbb{E}[\Delta(i)])$,

$$
\begin{aligned}
\frac{(\sqrt{p n / k+q n}+q \sqrt{n / k \log (n)}+\sqrt{\log (n)})}{\mathbb{E}[\Delta(i)]} & =\frac{O(\sqrt{\log (m)(\alpha+k \beta)})+O(\sqrt{\log (n)})}{\Omega((\alpha-\beta) \log (m))} \\
& =o(1) .
\end{aligned}
$$

Above we assumed that $k=o(\log (n))$. Following from the intuition above we prove Theorems 1.1 and 1.2 in the appendix which imply that our SDP is optimal.

In the Appendix (Section A.1) we present an experimental evaluation of the two SDPs considered in this section. The experiments corroborate Theorem 1.1 and also show that the SDP in (2) experimentally seems to have a similar recovery performance as the (stronger) SDP in (4) however we could only prove a suboptimal result about it. We leave the possible optimality of the SDP in (2) as an open question.

\section{Proof of the main theorem}

In this section we prove our main theorem, Theorem 2.2 about the SDP defined by (4). We restate the SDP here.

$$
\begin{array}{ll}
\max & A(G) \bullet Y \\
\text { s.t. } & \sum_{j} Y_{i j}+\sum_{j} Y_{j i}=2 n / k \\
& Y_{i i}=1 \quad(\forall i) \\
& Y_{i j} \geq 0 \quad(\forall i, j) \\
& Y \succcurlyeq 0 .
\end{array}
$$


Let $Y^{*}$ be the matrix corresponding to the hidden partition $P^{*}=\left\{P_{t}\right\}$, i.e. $Y^{*}[i, j]=1$ if $i, j$ belong to the same cluster and 0 otherwise. Let $O P T(G)$ be the optimal value in the above SDP. We will show that $Y^{*}$ is the unique solution to SDP (4) w.h.p as long as the conditions in Theorem 2.2 are satisfied. This would prove Theorem 2.2. Our proof will be based on a dual certificate. In that context consider the dual formulation of the above SDP which is the following

$$
\begin{array}{ll}
\min & \operatorname{Trace}(D)+(2 n / k) \sum_{i} x_{i} \\
\text { s.t. } & D+\sum_{i} x_{i}\left(R_{i}+C_{i}\right)-Z-A \succcurlyeq 0 .
\end{array}
$$

where $D$ is a diagonal matrix, $x_{i}$ are scalars, $Z$ is a non-negative symmetric matrix (corresponding to the $\geq 0$ constraints) with 0 in the diagonal entries, $R_{i}$ is the matrix with 1 in every entry of row $i$ and 0 otherwise, $C_{i}=R_{i}^{T}$ is the matrix with 1 in every entry of column $i$ and 0 otherwise and we write $A$ instead of $A(G)$ when there is no fear of confusion.

Let $D U A L(G)$ be the optimal value of the above dual program. We will first exhibit a valid dual solution $M^{*}=\left(D^{*},\left\{x_{i}^{*}\right\}, Z^{*}\right)$ which, with high probability, has dual objective value $\delta$ such that $A \bullet Y^{*}=\delta$. But since $A \bullet Y^{*} \leq O P T(G) \leq D U A L(G)$ (by weak duality) we get that $Y^{*}$ is an optimal solution to the above SDP. We will also show uniqueness via complementary slackness.

Before moving on further it will be convenient to introduce the following definition which we will be used in the proof later. We also encourage the reader to revisit the Notations section (Section 1.2) at this time as it would help with the reading of what follows.

Definition 3.1. Given a partition of $n$ vertices $\left\{P_{t}\right\}_{t=1}^{k}$ we define the vectors $\left\{v_{t}\right\}$ to be the indicator vectors of the clusters. We further define the following subspaces, which are perpendicular to each other, and partition $\mathbb{R}^{n}$.

- $\mathbb{R}_{k}$ : the subspace spanned by the vectors $\left\{v_{t}\right\}$, i.e. the subspace of vectors with equal values in each cluster,

- $\mathbb{R}_{n \mid k}$ : the subspace perpendicular to $\mathbb{R}_{k}$, i.e. the subspace where the sum on each cluster is equal to 0.

At this point it is useful to look at what the complementary slackness condition implies. Since strong duality holds in the case of our SDP (easy to check that Slater's conditions are satisfied) we have that complementary slackness is zero which implies that

$$
\operatorname{Trace}\left(M^{*} Y^{*}\right)=\operatorname{Trace}\left(M^{*} \sum v_{t} v_{t}^{T}\right)=0 .
$$

for any optimal dual solution $M^{*}$. The above condition implies that for any such $M^{*}$ (since $M^{*}$ is $\mathrm{PSD}$ ) it must be that the subspace $\mathbb{R}_{k}$ is an eigenspace with eigenvalue 0 which implies

$$
(\forall i, t) \delta_{i \rightarrow P_{t}}\left(M^{*}\right)=0 \text {. }
$$

Having established the conditions that must be satisfied by the optimal dual solution $M^{*}$, we describe our candidate dual solution

$$
\left(D^{*},\left\{x_{i}^{*}\right\}, Z^{*}\right)
$$


We begin by describing the choice of $Z^{*}$. If vertex $i$ and $j$ belong to the same cluster then $Z^{*}[i, j]=0$ otherwise

$$
Z^{*}[i, j]=\left(\frac{\delta_{\max }^{\text {out }}(i)}{n / k}-\frac{\delta_{i \rightarrow P(j)}}{n / k}\right)+\left(\frac{\delta_{\max }^{\text {out }}(j)}{n / k}-\frac{\delta_{j \rightarrow P(i)}}{n / k}\right)+\left(\frac{\delta_{P(j) \rightarrow P(i)}}{(n / k)(n / k)}-\min _{t_{1}, t_{2}} \frac{\delta_{P_{t_{1}} \rightarrow P_{t_{2}}}}{(n / k)(n / k)}\right) .
$$

It is easy to see that the matrix $Z^{*}$ is symmetric by noting that exchanging $j$ and $i$ in the above expression leads to the same value. Also to see that each entry of $Z^{*}$ is non-negative note that $Z^{*}[i, j]$ is the sum of non-negative terms. Having defined $Z^{*}$ as above we choose $x_{i}^{*}$ to be such that the condition given in Equation 8 holds for the non-diagonal blocks, yielding:

$$
x_{i}^{*}=\frac{\delta_{\max }^{\text {out }}(i)}{n / k}-\frac{1}{2} \min _{t_{1}, t_{2}} \frac{\delta_{P_{t_{1}} \rightarrow P_{t_{2}}}}{(n / k)(n / k)} .
$$

And finally we define $D^{*}$ to balance out the sum along the diagonal blocks from $A$ as well as the $x_{i}^{*}$.

$$
D^{*}[i, i]=\delta^{\text {in }}(i)-\delta_{\max }^{\text {out }}(i)-\sum_{j \in P(i)} \frac{\delta_{\max }^{\text {out }}(j)}{n / k}+\min _{t_{1}, t_{2}} \frac{\delta_{P_{t_{1}} \rightarrow P_{t_{2}}}}{n / k}
$$

Interestingly, this dual certificate construction seems to share some features with the one proposed by Awasthi et al. $\left[\mathrm{ABC}^{+} 15\right]$ for an SDP relaxation for k-means clustering. While we were not able to make a formal connection, it would be very interesting if the reason for the similarities was the existence of some type of canonical way of building certificates for clustering problems, we leave this for future investigations.

Now consider the objective for the dual program (7). It is easy to see that it is equal to

$$
\operatorname{Trace}\left(D^{*}\right)+2 n / k \sum_{i} x_{i}^{*}=\sum_{i} \delta^{i n}(i)=A(G) \bullet Y^{*}
$$

The following lemma the proof of which we provide in the Appendix in Section A.6 implies that the above mentioned solution is a valid dual solution, proving that $Y^{*}$ is an optimal solution to the above program (by weak duality).

Lemma 3.2. The matrix $M^{*}=D^{*}+\sum_{i} x_{i}^{*}\left(R_{i}+C_{i}\right)-A-Z^{*}$ (as defined above) is such that with probability $1-n^{-\Omega(1)}$, if the condition (5) is satisfied, then

$$
M^{*} \succeq 0
$$

It is easy to show using complementary slackness that $Y^{*}$ is indeed the unique optimal solution with high probability. For completeness we provide the proof in the Appendix in Section A.6.4

\section{Note about the Monotone Adversary}

In this section, we extend our result to the following semi random model considered in the paper of Feige and Kilian [FK01]. We first define a monotone adversary (we define it for the "homophilic" case). Given a graph $G$ and a partition $P=\left\{P_{i}\right\}$ a monotone adversary is allowed to take any of the following two actions on the graph:

- Arbitrarily remove edges across clusters, i.e. $(u, v)$ s.t. $P(u) \neq P(v)$. 
- Arbitrarily add edges within clusters, i.e. $(u, v)$ s.t. $P(u)=P(v)$.

Given a graph $G$ let $G_{a d v}$ be the resulting graph after the adversary's actions. The adversary is monotone in the sense that the set of the optimal multisections in $G_{a d v}$ contains the set of the optimal multisections in $G$. Let $B(G)$ be the number of edges cut in the optimal multisection. We

now consider the following semi-random model, where we first randomly pick a graph $G \sim \mathcal{G}_{p, q, k}$ and then the algorithm is given $G_{a d v}$ where the monotone adversary has acted on $G$. The following theorem shows that our algorithm is robust against such a monotone adversary:

Theorem 4.1. Given a graph $G_{a d v}$ generated by a semi-random model described above we have that with probability $1-o(1)$ the algorithm described in section 3 recovers the original (hidden) partition. The probability is over the randomness in the production of $G \sim \mathcal{G}_{p, q, k}$ on which the adverary acts.

We provide the proof of the above theorem in the Appendix in Section A.8

\section{References}

[ABC $\left.{ }^{+} 15\right]$ P. Awasthi, A. S. Bandeira, M. Charikar, R. Krishnaswamy, S Villar, and R. Ward. Relax, no need to round: integrality of clustering formulations. 6th Innovations in Theoretical Computer Science (ITCS 2015), 2015.

[ABH14] E. Abbe, A. S. Bandeira, and G. Hall. Exact recovery in the stochastic block model. Available online at arXiv:1405.3267 [cs.SI], 2014.

[AK97] N. Alon and N. Kahale. A spectral technique for coloring random 3-colorable graphs. SIAM J. Comput., 26(6):1733-1748, 1997.

[AKS98] N. Alon, M. Krivelevich, and B. Sudakov. Finding a large hidden clique in a random graph. In Proceedings of the Ninth Annual ACM-SIAM Symposium on Discrete Algorithms, 25-27 January 1998, San Francisco, California., pages 594-598, 1998.

[AS15] E. Abbe and C. Sandon. Community detection in general stochastic block models: fundamental limits and efficient recovery algorithms. Available online at arXiv:1503.00609 [math.PR], 2015.

[AV13] E. Arias-Castro and N. Verzelen. Community Detection in Random Networks. Available online at arXiv:1302.7099 [math.ST], 2013.

[Ban15] A. S. Bandeira. Random Laplacian matrices and convex relaxations. Available online at arXiv:1504.03987 [math.PR], 2015.

[BCLS84] T. N. Bui, S. Chaudhuri, F. T. Leighton, and M. Sipser. Graph bisection algorithms with good average case behavior. In 25th Annual Symposium on Foundations of Computer Science, West Palm Beach, Florida, USA, 24-26 October 1984, pages 181-192, 1984.

[BCS15] A. S. Bandeira, Y. Chen, and A. Singer. Non-unique games over compact groups and orientation estimation in cryo-em. Available at arXiv:1505.03840 [cs.CV], 2015. 
[BCSZ14] A. S. Bandeira, M. Charikar, A. Singer, and A. Zhu. Multireference alignment using semidefinite programming. 5th Innovations in Theoretical Computer Science (ITCS 2014), 2014.

[Bop87] R. B. Boppana. Eigenvalues and graph bisection: An average-case analysis. In Proceedings of the 28th Annual Symposium on Foundations of Computer Science, SFCS '87, pages 280-285, Washington, DC, USA, 1987. IEEE Computer Society.

[BvH15] A. S. Bandeira and R. v. Handel. Sharp nonasymptotic bounds on the norm of random matrices with independent entries. Annals of Probability, to appear, 2015.

[CK01] A. Condon and R. M. Karp. Algorithms for graph partitioning on the planted partition model. Random Struct. Algorithms, 18(2):116-140, 2001.

[CMM06] M. Charikar, K. Makarychev, and Y. Makarychev. Near-optimal algorithms for unique games. In Proceedings of the Thirty-eighth Annual ACM Symposium on Theory of Computing, STOC '06, pages 205-214, New York, NY, USA, 2006. ACM.

[CRV15] P. Chin, A. Rao, and V. Vu. Stochastic block model and community detection in the sparse graphs: A spectral algorithm with optimal rate of recovery. Available online at: arXiv:1501.05021, 2015.

[CX14] Y. Chen and J. Xu. Statistical-computational tradeoffs in planted problems and submatrix localization with a growing number of clusters and submatrices. Available online at arXiv:1402.1267 [stat.ML], 2014.

[DKMZ11] A. Decelle, F. Krzakala, C. Moore, and L. Zdeborová. Asymptotic analysis of the stochastic block model for modular networks and its algorithmic applications. Phys. Rev. E, 84:066106, Dec 2011.

[ER59] P. Erdös and A. Renyi. On random graphs. I. Publicationes Mathematicae, 1959.

[FJ95] A. M. Frieze and M. Jerrum. Improved approximation algorithms for max k-cut and max bisection. In Proceedings of the 4th International IPCO Conference on Integer Programming and Combinatorial Optimization, pages 1-13, London, UK, UK, 1995. Springer-Verlag.

[FK01] U. Feige and J. Kilian. Heuristics for semirandom graph problems. J. Comput. Syst. Sci., 63(4):639-671, 2001.

[HWX14] B. Hajek, Y. Wu, and J. Xu. Achieving Exact Cluster Recovery Threshold via Semidefinite Programming. Available online at arXiv:1412.6156 [stat.ML], 2014.

[HWX15] B. Hajek, Y. Wu, and J. Xu. Achieving Exact Cluster Recovery Threshold via Semidefinite Programming: Extensions. Available online at arXiv:1502.07738 [stat.ML], 2015.

[KNS09] R. Krauthgamer, J. Naor, and R. Schwartz. Partitioning graphs into balanced components. In Proceedings of the Twentieth Annual ACM-SIAM Symposium on Discrete Algorithms, SODA '09, pages 942-949, Philadelphia, PA, USA, 2009. Society for Industrial and Applied Mathematics. 
[Mas14] L. Massoulié. Community detection thresholds and the weak ramanujan property. In Symposium on Theory of Computing, STOC 2014, New York, NY, USA, May 31 June 03, 2014, pages 694-703, 2014.

[McS01] F. McSherry. Spectral partitioning of random graphs. In Proceedings of the 42Nd IEEE Symposium on Foundations of Computer Science, FOCS '01, pages 529-, Washington, DC, USA, 2001. IEEE Computer Society.

[MMV14] K. Makarychev, Y. Makarychev, and A. Vijayaraghavan. Constant factor approximation for balanced cut in the PIE model. In Symposium on Theory of Computing, STOC 2014, New York, NY, USA, May 31 - June 03, 2014, pages 41-49, 2014.

[MNS12] E. Mossel, J. Neeman, and A. Sly. Stochastic Block Models and Reconstruction. Available online at arXiv: 1202.1499, 2012.

[MNS13] E. Mossel, J. Neeman, and A. Sly. A proof of the block model threshold conjecture. Available online at arXiv:1311.4115, 2013.

[MNS14a] E. Mossel, J. Neeman, and A. Sly. Belief propagation, robust reconstruction and optimal recovery of block models. In Proceedings of The 27th Conference on Learning Theory, COLT 2014, Barcelona, Spain, June 13-15, 2014, pages 356-370, 2014.

[MNS14b] E. Mossel, J. Neeman, and A. Sly. Consistency thresholds for binary symmetric block models. Available online at arxiv:140\%.1591, 2014.

[Vu14] V. Vu. A simple SVD algorithm for finding hidden partitions. Available online at arXiv:1404.3918, 2014.

[YP14] S.-Y. Yun and A. Proutiere. Accurate community detection in the stochastic block model via spectral algorithms. Available online at arXiv:1412.7335, 2014.

\section{A Appendix}

\section{A.1 Experimental Evaluation}

In this section we present some experimental results on the SDPs presented above. For both of the SDPs we consider the case of $p=\alpha \frac{\log (m)}{m}$ and $q=\beta \frac{\log (m)}{m}$ with $k=3$ and $m=20$. We vary $\alpha$ and $\beta$ and for each pair of values we take 10 independent instances and the shade of grey in the square represents the fraction of instances for which the SDP was integral with lighter representing higher fractions of integrality. The red lines represent the curve we prove in our main theorem 1.1 i.e. $\sqrt{\alpha}-\sqrt{\beta}>1$.

Figure 1 corroborates our theorem 1.1 as for SDP in (4) we observe that experimentally the performance almost exactly mimics what we prove. For the other (possibly) weaker SDP in (2) we see in Figure 2 that the performance is almost similar to the stronger SDP however we were unable to prove it formally as discussed Section 2. We leave this as an open question to show that SDP in 2 is integral all the way down to the information theoretic threshold (i.e. $\sqrt{\alpha}-\sqrt{\beta}>1$ ). We observe from the experiments above that this indeed seems to be the case. 


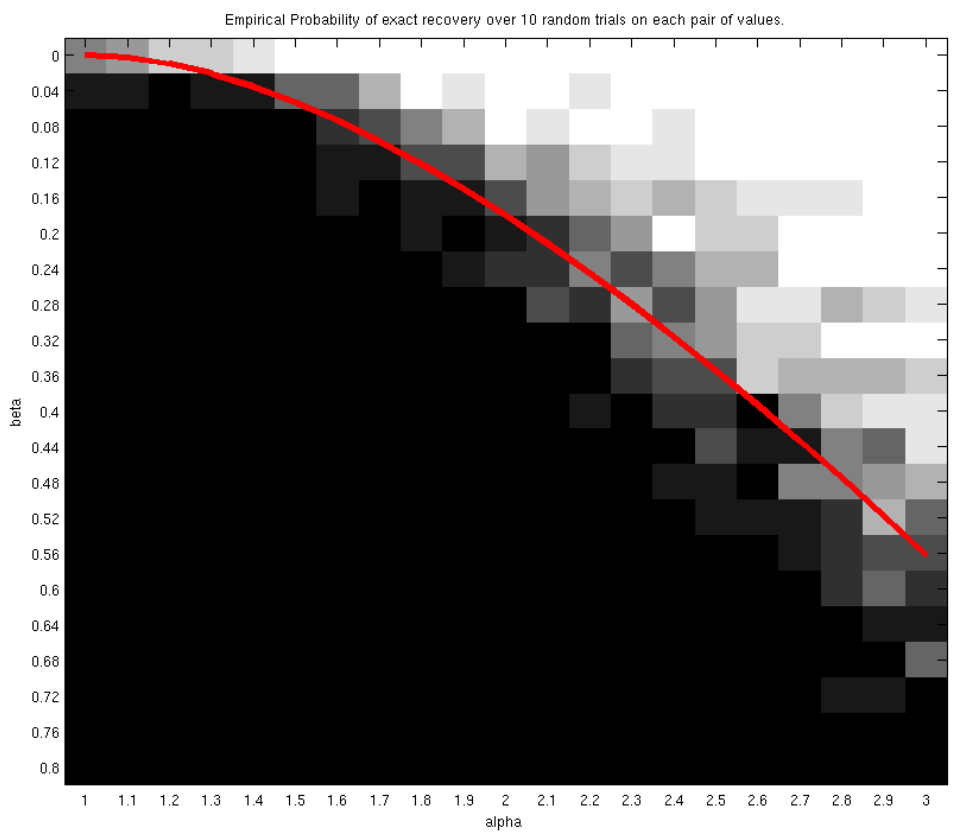

Figure 1: Performance of SDP in (4). We consider the case of $p=\alpha \frac{\log (m)}{m}$ and $q=\beta \frac{\log (m)}{m}$ with $k=3$ and $m=20$. We vary $\alpha$ and $\beta$ and for each pair of values we take 10 independent instances and the shade of grey in the square represents the fraction of instances for which the SDP was integral with lighter representing higher fractions of integrality. The red line represents the curve we prove in our main theorem 1.1 i.e. $\sqrt{\alpha}-\sqrt{\beta}>1$.

\section{A.2 The multireference alignment SDP for clustering}

In this section we describe an interesting connection between the SDPs used for clustering and partitioning problems and others such as ones used for the multireference signal alignment and the unique games problems.

For illustrative purposes we will consider a slightly different version of the balanced $k$-cut (multisection) problem described earlier. Instead of imposing that the graph is partitioned in equal sized clusters, we will consider the objective value to be maximized to be the difference between the number of agreeing pairs and disagreeing pairs where an agreeing pair is a pair of nodes connected by an edge that was picked to be in the same cluster or a pair of points not connected by an edge that is not in the same cluster, and disagreeing pairs are all the others. Note that, if the balanced partition constraint was enforced, this objective would be equivalent to the multisection one.

The multireference alignment problem in signal processing [BCSZ14] consists of aligning $n$ signals $y_{1}, \ldots, y_{n}$ with length $k$ that are copies of a single signal but have been shifted and corrupted with white gaussian noise. For $a \in[k]$, we set $R_{l_{i}}$ to be the $k \times k$ matrix that shifts the entries of vector by $a$ coordinates. In this notation, the maximum likelihood estimator for the multireference 


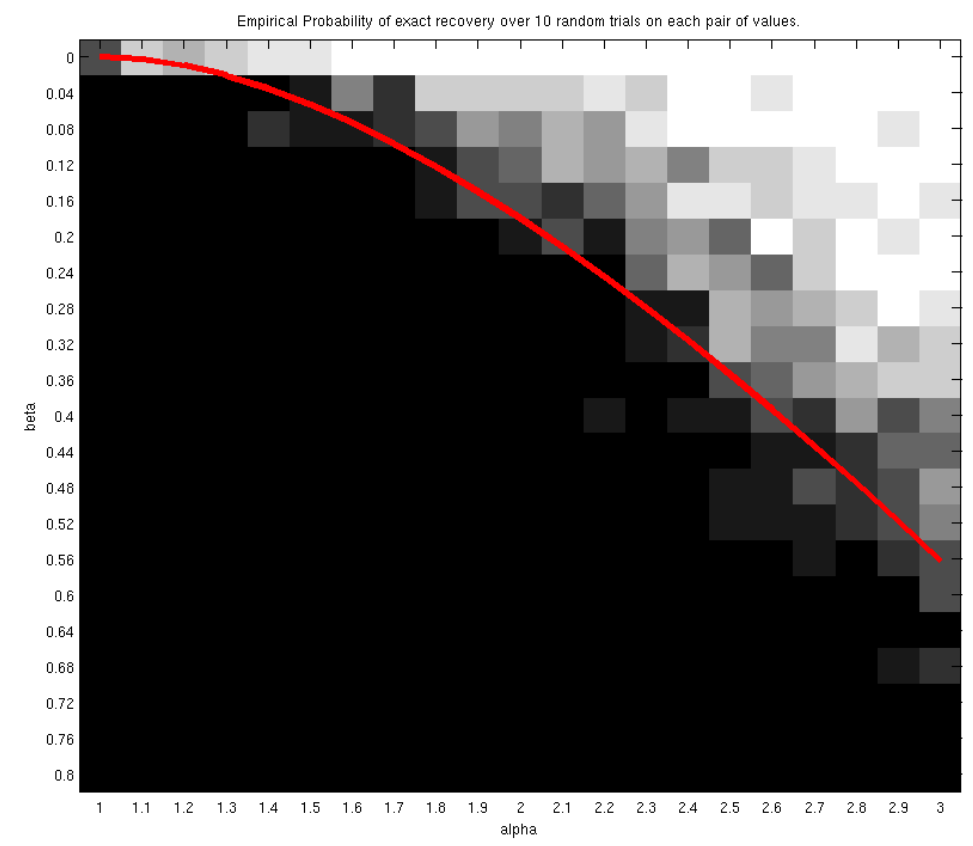

Figure 2: Performance of SDP in (2). We consider the case of $p=\alpha \frac{\log (m)}{m}$ and $q=\beta \frac{\log (m)}{m}$ with $k=3$ and $m=20$. We vary $\alpha$ and $\beta$ and for each pair of values we take 10 independent instances and the shade of grey in the square represents the fraction of instances for which the SDP was integral with lighter representing higher fractions of integrality. The red line represents the curve we prove in Theorem 1.1, for the $\operatorname{SDP}(4)$, i.e. $\sqrt{\alpha}-\sqrt{\beta}>1$.

alignment problem is given by the shifts $l_{1}, \ldots, l_{n} \in[k]$ that maximize

$$
\sum_{i, j=1}^{n}\left\langle R_{l_{i}}^{T} y_{i}, R_{l_{j}}^{T} y_{j}\right\rangle=\sum_{i, j=1}^{n} \operatorname{Tr}\left[y_{j} y_{i}^{T} R_{l_{i}} R_{l_{j}}^{T}\right] .
$$

A fruitful way of thinking about (9) is as a sum, over each pair $i, j$, of pairwise costs that depends on the choices of shifts for the variable in each pair. An example of a problem of this type is the celebrated Unique Games problem, and indeed the SDP approach developed in [BCSZ14] for the multireference alignment problem is an adaptation of an SDP based approximation algorithm for the Unique Games problems by Charikar et al. [CMM06]. The objective in the alignment problem (9) has, however, an important property - the pairwise costs only depends on the relative choices of shifts. More precisely, both $l_{i}$ and $l_{j}$ being increased by the same amount has no effect on the pairwise cost relative to $(i, j)$. In fact, there is a general framework for solving problems with this group invariance-type property, called non-unique games, when the group involved is compact [BCS15]. The example above and SDP (2) that we will derive below are particular cases of this framework, but it is more enlightening to derive the SDP we will use for partitioning from the multireference alignment one.

To obtain an SDP for the partitioning problem, one can think of each node $i$ as a signal $y_{i}$ in $\mathbb{R}^{k}$ and think of a shift label as a cluster membership, the cost associated to the pair $i, j$ should 
then: if the nodes are connected, +1 if the two signals are given the same shift and -1 otherwise; if the nodes are not connected it should be -1 if the two signals are given the same shift and +1 otherwise. This can be achieved by replacing $y_{j} y_{i}^{T}$ on the objective (9) by appropriate $k \times k$ matrices $C_{i j}^{T}=\frac{1}{k}\left(2 I-\mathbf{1 1}^{T}\right)$ if $i$ and $j$ are connected and $C_{i j}^{T}=\frac{1}{k}\left(\mathbf{1 1}^{T}-2 I\right)$ if not. Our objective would then be

$$
\sum_{a=1}^{k} \sum_{i, j \in \mathcal{C}_{a}} d_{i j}=-\sum_{i, j \in[n]} \operatorname{Tr}\left[C_{i j}^{T} R_{l_{i}} R_{l_{j}}^{T}\right],
$$

where $R_{l_{i}}$ is constrained to be a circulant permutation matrix (a shift operator).

The SDP relaxation proposed in [BCSZ14] would then take the form

$$
\begin{array}{ll}
\max & \operatorname{Tr}(C X) \\
\text { s. t. } & X_{i i}=I_{k \times k} \\
& X_{i j} \mathbf{1}=\mathbf{1} \\
& X_{i j} \text { is circulant } \\
& X \geq 0 \\
& X \succeq 0,
\end{array}
$$

It is clear, however, that (10) has many optimal solutions. Given an optimal selection of cluster labelings, any permutation of these labels will yield a solution with the same objective. For that reason we can adapt the SDP to consider the average of such solutions. This is achieved by restricting each block $X_{i j}$ to be a linear combination of $I_{k \times k}$ and $\mathbf{1 1}^{T}$ (meaning that it is constant both on the diagonal and on the off-diagonal). Adding that constraint yields the following SDP.

$$
\begin{array}{ll}
\max & \operatorname{Tr}(C X) \\
\text { s. t. } & X_{i i}=I_{k \times k} \\
& X_{i j} \mathbf{1}=\mathbf{1} \\
& X_{i j} \text { is circulant } \\
& \left(X_{i j}\right)_{a a}=\left(X_{i j}\right)_{11} \\
& \left(X_{i j}\right)_{a b}=\left(X_{i j}\right)_{12}, \quad \forall_{a \neq b} \\
& X \geq 0 \\
& X \succeq 0,
\end{array}
$$

Since the constraints in (11) imply

$$
\left(X_{i j}\right)_{11}+(k-1)\left(X_{i j}\right)_{12}=1,
$$

(11) can be described completely in terms of the variables $\left(X_{i j}\right)_{11}$. For that reason we consider the matrix $Z \in \mathbb{R}^{n \times n}$ with entries $Z_{i j}=\left(X_{i j}\right)_{11}$. We can then rewrite (11) as

$$
\begin{array}{ll}
\max & \operatorname{Tr}(\tilde{C} Z) \\
\text { s. t. } & Z_{i i}=1 \\
& Z \geq 0 \\
& Z^{(k)} \succeq 0,
\end{array}
$$

where $\tilde{C}_{i j}=k C_{i j}$ and $Z^{(k)}$ is the $n k \times n k$ matrix whose $n \times n$ diagonal blocks are equal to $Z$ and 
whose $n \times n$ non-diagonal blocks are equal to $\frac{11^{T}-Z}{k-1}$. For example,

$$
Z^{(2)}=\left[\begin{array}{cc}
Z & 11^{T}-Z \\
11^{T}-Z & Z
\end{array}\right] \text { and } Z^{(3)}=\left[\begin{array}{ccc}
Z & \frac{11^{T}-X}{2} & \frac{11^{T}-Z}{12} \\
\frac{11^{T}-Z}{2} & Z & \frac{11^{T^{2}}-Z}{2} \\
\frac{11^{T}-Z}{2} & \frac{11^{T}-Z}{2} & Z
\end{array}\right] .
$$

The following lemma gives a simpler characterization for the intriguing $Z^{(k)} \succeq 0$ constraint.

Lemma A.1. Let $Z$ be a symmetric matrix and $k \geq 2$ an integer. $Z^{(k)} \succeq 0$ if and only if $Z \succeq \frac{1}{k} \mathbf{1 1}^{T}$.

Before proving Lemma A.1 we note that it implies that we can succinctly rewrite (12) as

$$
\begin{array}{ll}
\max & \operatorname{Tr}(\tilde{C} Z) \\
\text { s.t. } & Z_{i i}=1 \\
& Z \geq 0 \\
& Z \succeq \frac{1}{k} \mathbf{1 1}^{T} .
\end{array}
$$

A simple change of variables $Y=\frac{k}{k-1} Z-\frac{1}{k-1} \mathbf{1 1}^{T}$, allows one to rewrite (13) as (for appropriate matrix $C^{\prime}$ and constant $c^{\prime}$ ),

$$
\begin{array}{ll}
\max & \operatorname{Tr}\left(C^{\prime} Y\right)-c^{\prime} \\
\text { s. t. } & Y_{i i}=1 \\
& Y_{i j} \geq-\frac{1}{k-1} \\
& Y \succeq 0 .
\end{array}
$$

Remarkably,(14) coincides with the classical semidefinite relaxation for the Max-k-Cut problem [FJ95], which corresponds to (2) used in this paper.

Proof. [of Lemma A.1]

Since, in this proof, we will be using 1 to refer to the all-ones vector in two different dimensions we will include a subscript denoting the dimension of the all-ones vector.

The matrix $Z^{(k)}$ is block circulant and so it can be block-diagonalizable by a block DFT matrix, $F_{k \times k} \otimes I_{n \times n}$, where $F_{k \times k}$ is the $k \times k$ (normalized) DFT matrix and $\otimes$ is the Kronecker product. In other words,

$$
\left(F_{k \times k} \otimes I_{n \times n}\right) Z^{(k)}\left(F_{k \times k} \otimes I_{n \times n}\right)^{T}
$$

is block diagonal. Furthermore, note that

$$
Z^{(k)}=\left(\mathbf{1}_{k} \mathbf{1}_{k}^{T} \otimes \frac{\mathbf{1}_{n} \mathbf{1}_{n}^{T}-Z}{k-\mathbf{1}}\right)-\left(I_{k \times k} \otimes\left[Z-\frac{\mathbf{1}_{n} \mathbf{1}_{n}^{T}-Z}{k-1}\right]\right) .
$$

Also, It is easy to check that

$$
\left(F_{k \times k} \otimes I_{n \times n}\right)\left(I_{k \times k} \otimes\left[Z-\frac{\mathbf{1}_{n} \mathbf{1}_{n}^{T}-Z}{k-1}\right]\right)\left(F_{k \times k} \otimes I_{n \times n}\right)^{T}=I_{k \times k} \otimes\left[Z-\frac{\mathbf{1}_{n} \mathbf{1}_{n}^{T}-Z}{k-1}\right],
$$

and

$$
\left(F_{k \times k} \otimes I_{n \times n}\right)\left(\mathbf{1}_{k} \mathbf{1}_{k}^{T} \otimes \frac{\mathbf{1}_{n} \mathbf{1}_{n}^{T}-Z}{k-1}\right)\left(F_{k \times k} \otimes I_{n \times n}\right)^{T}=k\left(e_{1} e_{1}^{T} \otimes \frac{\mathbf{1}_{n} \mathbf{1}_{n}^{T}-Z}{k-1}\right),
$$


This means that $\left(F_{k \times k} \otimes I_{n \times n}\right) Z^{(k)}\left(F_{k \times k} \otimes I_{n \times n}\right)^{T}$ is a block diagonal matrix with the first block equal to $\mathcal{A}$ and all other diagonal blocks equal to $\mathcal{B}$ where $\mathcal{A}$ and $\mathcal{B}$ are given by

$$
\mathcal{A}=Z-\frac{\mathbf{1}_{n} \mathbf{1}_{n}^{T}-Z}{k-1}+k \frac{\mathbf{1}_{n} \mathbf{1}_{n}^{T}-Z}{k-1}=\mathbf{1}_{n} \mathbf{1}_{n}^{T} \text { and } \mathcal{B}=Z-\frac{\mathbf{1}_{n} \mathbf{1}_{n}^{T}-Z}{k-1} .
$$

Thus, the condition $Z^{(k)} \succeq 0$ is equivalent to $Z-\frac{\mathbf{1}_{n} \mathbf{1}_{n}^{T}-Z}{k-1} \succeq 0$ which can be rewritten as,

$$
Z-\frac{1}{k} \mathbf{1}_{n} \mathbf{1}_{n}^{T} \succeq 0
$$

\section{A.3 Proof of Optimality - Theorem 1.2}

Proof. The theorem follows directly from the lower bound presented in [ABH14]. They showed that [ABH14, Theorem 1] when we sample $G \sim G_{p, q, 2}$ with $p=\alpha^{\prime} \frac{\log (n)}{n}$ and $q=\beta^{\prime} \frac{\log (n)}{n}$, it is information theoretically impossible to correctly recover the clusters with high probability if

$$
\sqrt{\alpha^{\prime}}-\sqrt{\beta^{\prime}}<\sqrt{2}
$$

Now consider $G \sim G_{p, q, k}$ with $p=\alpha \frac{\log (m)}{m}$ and $q=\beta \frac{\log (m)}{m}$. Suppose that the algorithm was given the membership of vertices in all the clusters except two of them. A direct application of the above theorem yields that it is information theoretically impossible to correctly recover the two unrevealed clusters with high probability if

$$
\sqrt{2 \frac{\log (m)}{\log (n)}}(\sqrt{\alpha}-\sqrt{\beta})<\sqrt{2}
$$

which is equivalent to

$$
\sqrt{\alpha}-\sqrt{\beta}<\frac{\log (n)}{\log (m)}=1+\frac{\log (k)}{\log (m)}=1+o_{n}(1)
$$

which proves the bound.

\section{A.4 Proof of Optimality - Theorem 1.1}

Proof. We will use the condition of theorem 1.3 and the following lemma, to prove theorem 1.1.

Lemma A.2. Let $p=\frac{\alpha \log (m)}{m}$ and $q=\frac{\beta \log (m)}{m}$. Let $k=\gamma \log (m)$ (where $\left.\gamma=O(1)\right)$. Now we have that as long as

$$
\sqrt{\alpha}-\sqrt{\beta}>\sqrt{1+c_{1} \sqrt{\beta \gamma}\left(1+\log \left(\sqrt{\frac{\alpha}{\beta}}\right)\right)}
$$

then for sufficiently large $n$ we have that with probability at least $1-n^{-\Omega(1)} \forall i, t$

$$
\delta^{i n}(i)-\delta_{i \rightarrow P_{t}}>c_{2}(\sqrt{\beta \gamma} \log (n)+\sqrt{\alpha \log (n)})
$$

where $c_{2}>0$ be any fixed number and $c_{1}>0$ in (15) is a constant depending on $c_{2}$ 
To complete the proof of theorem 1.1 we first observe that for the given range of parameters $p=\frac{\alpha \log (m)}{m}$ and $q=\frac{\beta \log (m)}{m}$ condition (5) in Theorem 1.3 becomes

$$
\hat{c}\left(\sqrt{p n / k+q n}+q \sqrt{\frac{n}{k} \log (n)}+\sqrt{\log (n)}+\log (k)\right) \leq c_{2}(\sqrt{\beta k \log (m)}+\sqrt{\alpha \log (n)})
$$

However, Lemma A.2 implies that with probability $1-n^{-\Omega(1)}$ we have that if condition 15 is satisfied then $\forall i, t$

$$
\delta^{i n}(i)-\delta_{i \rightarrow P_{t}}>c_{2}(\sqrt{\beta \gamma} \log (n)+\sqrt{\alpha \log (n)})
$$

where $c_{2}>0$ depends on $\hat{c}$. Therefore with probability $1-n^{-\Omega(1)}$ the condition in (5) of Theorem 1.3 is satisfied which in turn implies the SDP in Theorem 1.3 recovers the clusters, which concludes the proof of Theorem 1.1. Note that setting $\gamma=o(1)$ we get the case $k=o(\log (n))$ and the above condition reduces to $\sqrt{\alpha}-\sqrt{\beta}>1+o_{n}(1)$.

In the rest of the section we prove Lemma A.2. For the remainder of this section we borrow the notation from Abbe et al. [ABH14]. In [ABH14, Definition 3, Section A.1], they define the following quantity $T(m, p, q, \delta)$ which we use:

Definition A.3. Let $m$ be a natural number, $p, q \in[0,1]$, and $\delta \geq 0$, define

$$
T(m, p, q, \delta)=\mathbb{P}\left[\sum_{i=1}^{m}\left(Z_{i}-W_{i}\right) \geq \delta\right],
$$

where $W_{i}$ are i.i.d $\operatorname{Bernoulli}(p)$ and $Z_{i}$ are i.i.d. Bernoulli $(q)$, independent of the $W_{i}$.

Let $Z=\sum_{i=1}^{m} Z_{i}$ and $W=\sum_{i=1}^{m} W_{i}$. The proof is similar to proof of [ABH14, Lemma 8 , Section A.1] with modifications.

Proof. (of Lemma A.2) We will bound the probability of the bad event

$$
\delta^{i n}(i)-\delta_{i \rightarrow P_{t}} \leq c_{2}(\sqrt{\beta \gamma} \log (n)+\sqrt{\alpha \log (n)}) .
$$

Note that $\delta_{i n}(i)$ is a binomial variable with parameter $p$ and similarly $\delta_{i \rightarrow P_{t}}$ is a binomial variable with parameter $q$ and therefore, following the notation of [ABH14], we have that the probability of this bad event is

$$
T\left(m, p, q,-c_{2}(\sqrt{\beta \gamma} \log (n)+\sqrt{\alpha \log (n)})\right) .
$$

We show the following strengthening of their lemma.

Lemma A.4. Let $W_{i}$ be a sequence of i.i.d Bernoulli $\left(\frac{\alpha \log (m)}{m}\right)$ random variables and $Z_{i}$ an independent sequence of i.i.d Bernoulli $\left(\frac{\beta \log (m)}{m}\right)$ random variables, then the following bound holds for $m$ sufficiently large:

$$
\begin{aligned}
T\left(m, \frac{\alpha \log (m)}{m}, \frac{\beta \log (m)}{m},-c_{2}(\sqrt{\beta \gamma} \log (n)+\sqrt{\alpha \log (n)})\right) \leq \\
\quad \exp \left(-\left(\alpha+\beta-2 \sqrt{\alpha \beta}-c_{1} \sqrt{\beta \gamma}\left(1+\log \left(\sqrt{\frac{\alpha}{\beta}}\right)\right)+o(1)\right) \log (m)\right)
\end{aligned}
$$

where $c_{2}>0$ is a fixed number and $c_{1}>0$ depends only on $c_{2}$. 
Assuming the above lemma and taking a union bound over all clusters and vertices we get the following sequence of equations which proves Theorem A.2

$$
\begin{aligned}
& \mathbb{P}\left((\exists i, t) \delta^{i n}(i)-\delta_{i \rightarrow P_{t}} \leq c_{2}(\sqrt{\beta \gamma} \log (n)+\sqrt{\alpha \log (n)})\right) \\
& \leq m k^{2} \exp \left(-\left(\alpha+\beta-2 \sqrt{\alpha \beta}-c_{1} \sqrt{\beta \gamma}\left(1+\log \left(\sqrt{\frac{\alpha}{\beta}}\right)\right)+o(1)\right) \log (m)\right) \\
& \leq \exp \left(-\left(\alpha+\beta-2 \sqrt{\alpha \beta}-1-c_{1} \sqrt{\beta \gamma}\left(1+\log \left(\sqrt{\frac{\alpha}{\beta}}\right)\right)+o(1)\right) \log (m)\right) \\
& \leq m^{-\Omega(1)} \\
& \leq n^{-\Omega(1)}
\end{aligned}
$$

Proof of Lemma A.4. The proof of lemma A.4 is a simple modification of the proof of [ABH14, Lemma 8, Section A.1]. We mention the proof here for completeness.

Define $r=c_{2}(\sqrt{\beta \gamma} \log (n)+\sqrt{\alpha \log (n)}) \leq c_{1} \sqrt{\beta \gamma} \log (n)$ (for some fixed $c_{1}>0$ depending only on $c_{2}$ ) and let $Z=\sum Z_{i}$ and $W=\sum W_{i}$. We split $T$ as follows:

$$
T(m, p, q,-r)=\mathbb{P}\left(-r \leq Z-W \leq \log ^{2}(m)\right)+\mathbb{P}\left(Z-W \geq \log ^{2}(m)\right) .
$$

Lets bound the second term first. A simple application of Bernstein's Inequality (the calculations are shown in [ABH14, Lemma 8, Section A.1]) shows that Therefore we have that

$$
\mathbb{P}\left(Z-W \geq \log ^{2}(m)\right) \leq \exp \left(-\Omega(1) \frac{\log ^{2}(m)}{\log (\log (m))}\right) .
$$

We now bound the first term $\mathbb{P}\left(-r \leq Z-W \leq \log ^{2}(m)\right)$. Define

$$
\hat{r}=\operatorname{argmax}_{x} \mathbb{P}(Z-W=-x)
$$

Now it is easy to see that $\hat{r}=O(\log (m))$ (for $p=\alpha \frac{\log (m)}{m}$ and $\left.q=\beta \frac{\log (m)}{m}\right)$. Let $r_{\text {max }}=\max (r, \hat{r})$ and $r_{\min }=\min (r, \hat{r})$.

$$
\begin{aligned}
\mathbb{P}\left(-r \leq Z-W \leq \log ^{2}(m)\right) \leq & \left(\log ^{2}(m)+r_{\text {max }}\right) \mathbb{P}\left(Z-W=-r_{\text {min }}\right) \\
\leq & \left(\log ^{2}(m)+r_{\text {max }}\right)\left(\sum_{k_{2}=r_{\text {min }}}^{\log ^{2}(m)+r_{\text {max }}} \mathbb{P}\left(Z=k_{2}-r\right) \mathbb{P}\left(W=k_{2}\right)\right. \\
+\sum_{k_{2}=\log ^{2}(m)+r_{\text {min }}}^{m} & \left.\mathbb{P}\left(Z=k_{2}-r\right) \mathbb{P}\left(W=k_{2}\right)\right) \\
\leq & \left(\log ^{2}(m)+r_{\text {max }}\right)^{2} \max _{k_{2}}\left\{\mathbb{P}\left(Z=k_{2}-r_{\text {min }}\right) \mathbb{P}\left(W=k_{2}\right)\right\} \\
& +\left(\log ^{2}(m)+r_{\text {max }}\right) \mathbb{P}\left(Z \geq \log ^{2}(n)\right) \mathbb{P}\left(W \geq \log ^{2}(m)\right)
\end{aligned}
$$


The first inequality follows easily from considering both the cases $\hat{r} \geq r$ or $\hat{r} \leq r$. Similar probability estimates (using Bernstein) as before give that both

$$
\mathbb{P}\left(Z \geq \log ^{2}(m)\right), \mathbb{P}\left(W \geq \log ^{2}(m)\right) \leq \exp \left(-\Omega(1) \frac{\log (m)}{\log (\log (m))}\right)
$$

We now need to bound $\max _{k_{2}}\left\{\mathbb{P}\left(Z=k_{2}-r\right) \mathbb{P}\left(W=k_{2}\right)\right\}$ for which we use Lemma A.5 which is a modification of $[\mathrm{ABH} 14$, Lemma 7, Section A.1]. Plugging the estimates from above and noting that $\max _{k_{2}}\left\{\mathbb{P}\left(Z=k_{2}-r\right) \mathbb{P}\left(W=k_{2}\right)\right\}=T^{*}\left(m, p, q, \frac{r_{\min }}{\log (m)}\right)$ (defined in Lemma A.5) we get that

$\mathbb{P}\left(-r \leq Z-W \leq \log ^{2}(m)\right) \leq O\left(\log ^{4}(n)\right) T^{*}\left(m, p, q, \frac{r_{\min }}{\log (m)}\right)+\log ^{2}(n) \exp \left(-\Omega(1) \frac{\log (m)}{\log (\log (m))}\right)$

Putting everything together we get that

$T(m, p, q, 0) \leq 2 \log ^{4}(n) T^{*}\left(m, p, q, \frac{r_{\text {min }}}{\log (m)}\right)+\log ^{2}(n) \exp \left(-\Omega(1) \frac{\log (m)}{\log (\log (m))}\right)+\exp \left(-\Omega(1) \frac{\log (m)}{\log (\log (m))}\right)$

Using Lemma A.5 it follows from the above equation that

$$
\begin{aligned}
-\log (T(m, p, q,-r)) & \geq-\Omega(\log (\log (m)))+g\left(\alpha, \beta, \frac{r_{\min }}{\log (n)}\right) \log (m)-o(\log (m)) \\
& \geq\left(\alpha+\beta-2 \sqrt{\alpha \beta}-c_{1} \sqrt{\beta} \gamma\left(1+\log \left(\sqrt{\frac{\alpha}{\beta}}\right)\right)\right) \log (m)-o(\log (m))
\end{aligned}
$$

For the first inequality we use Lemma A.5 and set $\epsilon=\frac{r_{\min }}{\log (n)}$. For the second inequality we use the fact that $\epsilon \leq c_{1} \sqrt{\beta \gamma}$.

Lemma A.5. Let $p=\frac{\alpha \log (m)}{m}$ and $q=\frac{\beta \log (m)}{m}$ and let $W_{i}$ be a sequence of i.i.d Bernoulli- $p$ random variables and $Z_{i}$ an independent sequence of i.i.d Bernoulli-q random variables. Define

$$
\begin{aligned}
& V^{\prime}(m, p, q, \tau, \epsilon)=\mathbb{P}\left(\sum Z_{i}=\tau \log (m)\right) \mathbb{P}\left(\sum W_{i}=(\tau+\epsilon) \log (m)\right) \\
& =\left(\begin{array}{c}
m \\
\tau \log (m)
\end{array}\right) q^{\tau \log (m)}(1-q)^{m-\tau \log (m)}\left(\begin{array}{c}
m \\
(\tau+\epsilon) \log (m)
\end{array}\right) p^{(\tau+\epsilon) \log (m)}(1-p)^{m-(\tau+\epsilon) \log (m)},
\end{aligned}
$$

where $\epsilon=O(1)$. We also define the function

$$
g(\alpha, \beta, \epsilon)=(\alpha+\beta)-\epsilon \log (\alpha)-2 \sqrt{\left(\frac{\epsilon}{2}\right)^{2}+\alpha \beta}+\frac{\epsilon}{2} \log \left(\alpha \beta \frac{\sqrt{\left(\frac{\epsilon}{2}\right)^{2}+\alpha \beta}+\frac{\epsilon}{2}}{\sqrt{\left(\frac{\epsilon}{2}\right)^{2}+\alpha \beta}-\frac{\epsilon}{2}}\right) .
$$

Then we have the following results for $T^{*}(m, p, q, \epsilon)=\max _{\tau>0} V^{\prime}(m, p, q, \tau, \epsilon):$ for $m \in N$ and $\forall \tau>0$

$$
-\log \left(T^{*}(m, p, q, \epsilon)\right) \geq \log (m) g(\alpha, \beta, \epsilon)-o(\log (m)) .
$$

Proof. The proof of the above lemma is computational and follows from the carefully bounding the combinatorial coefficients. Note that

$$
\begin{aligned}
\log (V(m, p, q, \tau, \epsilon))=\quad & \log \left(\begin{array}{c}
m \\
\tau \log (m)
\end{array}\right)+\log \left(\begin{array}{c}
m \\
(\tau+\epsilon) \log (m)
\end{array}\right)+\tau \log (m) \log (p q)+ \\
& \epsilon \log (m) \log \left(\frac{p}{1-p}+(m-\tau \log (m)) \log ((1-p)(1-q))\right)
\end{aligned}
$$


Substituting the values of $p$ and $q$ we get

$$
\begin{aligned}
\log (V(m, p, q, \tau, \epsilon))=\quad & \log \left(\begin{array}{c}
m \\
\tau \log (m)
\end{array}\right)+\log \left(\begin{array}{c}
m \\
(\tau+\epsilon) \log (m)
\end{array}\right) \\
& +\tau \log (m)(\log (\alpha \beta)+2 \log \log (m)-2 \log (m)) \\
& +\epsilon \log (m)\left(\log (\alpha)+\log \log (m)-\log (m)+\alpha \frac{\log (m)}{m}\right) \\
& -\log (m)(\alpha+\beta)+o(\log (m))
\end{aligned}
$$

We now use the following easy inequality

$$
\log \left(\begin{array}{l}
n \\
k
\end{array}\right) \leq k(\log (n e)-\log (k))
$$

and now replacing this in the above equation gives us

$$
\begin{array}{r}
-\log (V(m, p, q, \tau, \epsilon)) \geq \log (m)\left((\alpha+\beta)+(\tau+\epsilon) \log \left(\frac{\tau+\epsilon}{e}\right)+\tau \log \left(\frac{\tau}{\epsilon}\right)-\tau \log (\alpha \beta)-\epsilon \log (\alpha)\right) \\
-o(\log (m))
\end{array}
$$

Now optimizing over $\tau$ proves the lemma.

\section{A.5 Proofs of Lemmas for the SDP in (4)}

\section{A.6 Proof of lemma 3.2}

We remind the reader that the proof of the lemma below continues the use of the notation used in Section 3

Proof. To prove this lemma we first show that Equation 8 is satisfied for $M^{*}$. This implies that the vectors $\left\{v_{t}\right\}$ which are indicator vectors for the clusters are an eigenvector with eigenvalue 0 . Consider the value of $\delta_{i \rightarrow P_{t}}\left(M^{*}\right)$ when $P_{t}=P(i)$. In this case

$$
\begin{aligned}
\delta_{i \rightarrow P_{t}}\left(M^{*}\right) & =D^{*}[i, i]+\frac{n}{k} x_{i}^{*}+\sum_{i^{\prime} \in P(i)} x_{i^{\prime}}^{*}-\sum_{i^{\prime} \in P(i)} A\left[i, i^{\prime}\right] \\
& =0 .
\end{aligned}
$$

where the last equality follows directly from the definitions of the dual certificate. Now consider the value of $\delta_{i \rightarrow P_{t}}\left(M^{*}\right)$ when $P_{t} \neq P(i)$. In this case 


$$
\begin{aligned}
\delta_{i \rightarrow P_{t}}\left(M^{*}\right)= & \frac{n}{k} x_{i}^{*}+\sum_{j \in P_{t}} x_{j}^{*}-\sum_{j \in P_{t}}(Z[i, j]+A[i, j]) \\
= & \frac{n}{k} x_{i}^{*}+\sum_{j \in P_{t}} x_{j}^{*}-\sum_{j \in P_{t}}\left(\frac{\delta_{\max }^{\text {out }}(i)}{n / k}+\frac{\delta_{\max }^{\text {out }}(j)}{n / k}-\left(\frac{\delta_{i \rightarrow P(j)}}{n / k}+A[i, j]\right)+\right. \\
& \left.\left(-\frac{\delta_{j \rightarrow P(i)}}{n / k}+\frac{\delta_{P(j) \rightarrow P(i)}}{(n / k)(n / k)}\right)-\min _{t_{1}, t_{2}} \frac{\delta_{P_{t_{1}} \rightarrow P_{t_{2}}}}{(n / k)(n / k)}\right) \\
= & \frac{n}{k} x_{i}^{*}+\sum_{j \in P_{t}} x_{j}^{*}-\sum_{j \in P_{t}}\left(\frac{\delta_{\max }^{\text {out }}(i)}{n / k}+\frac{\delta_{\max }^{\text {out }}(j)}{n / k}-\min _{t_{1}, t_{2}} \frac{\delta_{P_{t_{1}} \rightarrow P_{t_{2}}}}{(n / k)(n / k)}\right) \\
= & 0 .
\end{aligned}
$$

The third equality follows by noting that the terms in the parenthesis in the expression in the second line go to zero in summation. The fourth equality follows directly from the definitions.

The above implies that for all $t, M^{*} v_{t}=0$. Therefore we only need to show that $M^{*}$ is PSD with high probability on the subspace $\mathbb{R}_{n \mid k}$ (which is perpendicular to $\mathbb{R}_{k}=\operatorname{span}\left(\left\{v_{k}\right\}\right)$ ). To that end, note that if a matrix $W$ is such that for all $i, W\left[i, j_{1}\right]=W\left[i, j_{2}\right]$ when $P\left(j_{1}\right)=P\left(j_{2}\right)$ then for any $x \in \mathbb{R}_{n \mid k}, W x=0$, and similarly if for all $j, W\left[i_{1}, j\right]=W\left[i_{2}, j\right]$ when $P\left(i_{1}\right)=P\left(i_{2}\right)$ then for any $x \in \mathbb{R}_{n \mid k}, x^{T} W=0$. Therefore we have that $x^{T} Z^{*} x=x^{T}\left(R_{i}+C_{i}\right) x=0$ and so $x^{T} M^{*} x=x^{T} D^{*} x-x^{T} A x$.

In order to finish the proof it is enough to show that for all $x \in \mathbb{R}_{n \mid k}$

$$
x^{T}\left(D^{*}-A\right) x \geq 0 .
$$

In order to prove the above equation, and conclude the proof of Theorem 2.2 we use the following two lemmas, which we prove in the Appendix.

Lemma A.6. Define $\lambda_{\max }(A(G))$ to be the maximum over all $x \in \mathbb{R}_{n \mid k}$ of $x^{T} A(G) x$. With probability $1-n^{-\Omega(1)}$ over the choice of $G, \lambda_{\max }(A(G))$ is bounded by

$$
\lambda_{\max }(A(G)) \leq 3 \sqrt{p n / k+q n}+c \sqrt{\log (n)} .
$$

where $c$ is a universal constant.

Lemma A.7. With probability $1-n^{-\Omega(1)}$ we have that for all clusters $P_{t}$

$$
\sum_{j \in P_{t}} \frac{\delta_{\max }^{\text {out }}(j)}{n / k} \leq \frac{q n}{k}+30\left(\sqrt{\frac{n \log (k)}{k}} q+\log (k)+\sqrt{\frac{n}{k} \log (n)} \cdot \max \left\{q, \sqrt{\frac{q \log (n)}{n / k}}, \frac{\log (n)}{n / k}\right\}\right)
$$

and for all pairs of clusters $P_{t_{1}}$ and $P_{t_{2}}$

$$
\min _{t_{1}, t_{2}} \frac{\delta_{P_{t_{1}} \rightarrow P_{t_{2}}}}{n / k} \geq \frac{q n}{k}-2 \sqrt{q \log (n)} .
$$


Using those two lemmas, we can now conclude the proof of Theorem 2.2 as follows: We separate $D^{*}=D_{1}^{*}-D_{2}^{*}$, where $D_{1}^{*}, D_{2}^{*}$ are diagonal matrices

$$
\begin{aligned}
& D_{1}^{*}[i, i]=\delta^{\text {in }}(i)-\delta_{\max }^{\text {out }}(i) \\
& D_{2}^{*}[i, i]=\sum_{j \in P(i)} \frac{\delta_{\max }^{\text {out }}(j)}{n / k}-\min _{t_{1}, t_{2}} \frac{\delta_{P_{t_{1}} \rightarrow P_{t_{2}}}}{n / k} .
\end{aligned}
$$

Now for any $x \in \mathbb{R}_{n \mid k}$ lets consider $x^{T}\left(D^{*}-A\right) x$

$$
\begin{aligned}
x^{T}\left(D^{*}-A\right) x \geq & \min _{i} D_{1}^{*}[i, i]-\left(\max _{i} D_{2}^{*}[i, i]+\max _{x \in \mathbb{R}_{n \mid k}} x^{T} A x\right) \\
\geq & \min _{i} D_{1}^{*}[i, i]-\left(30\left(\sqrt{\frac{n \log (k)}{k}} q+\log (k)+\sqrt{\frac{n}{k} \log (n)} \cdot \max \left\{q, \sqrt{\frac{q \log (n)}{n / k}}, \frac{\log (n)}{n / k}\right\}\right)\right. \\
& +3 \sqrt{p n / k+q n}+c \sqrt{\log (n)}) \\
\geq & \min _{i} D_{1}^{*}[i, i]-\hat{c}\left(\sqrt{p n / k+q n}+q \sqrt{\frac{n}{k} \log (n)}+\sqrt{\log (n)}+\log (k)\right) \\
\geq & 0 .
\end{aligned}
$$

where $\hat{c}$ is a universal constant. The second inequality follows by direct substitutions from Equations $18,19,20$, the third inequlity follows from noting that $n$ is large enough such that $\sqrt{q n}>>\sqrt{q} \log (n)$ and $\sqrt{\log (n)} \frac{\log (n)}{\sqrt{\frac{n}{k}}}<<\sqrt{\log (n)}$ and $\sqrt{q n \frac{\log (k)}{k}} \leq \sqrt{q n}$. The last inequality follows from condition 5 of Theorem 2.2 .

\section{A.6.1 Proof of Lemma A.6}

We use the following recent sharp concentration result [BvH15, Corollary 3.12].

Theorem A.8 (Bandeira et al. [BvH15]). Let $X$ be an $n \times n$ symmetric matrix whose entries $X_{i j}$ are independent centered random variables. Then there exists for any $0<\epsilon \leq 1 / 2$ a universal constant $\tilde{c_{\epsilon}}$ such that for every $t \geq 0$

$$
\mathbb{P}(|X| \geq(1+\epsilon) 2 \sqrt{2} \tilde{\sigma}+t) \leq n e^{-t^{2} / \tilde{\epsilon_{\epsilon}} \sigma_{*}^{2}}
$$

where

$$
\tilde{\sigma}=\max _{i} \sqrt{\sum_{j} \mathbb{E}\left[X_{i j}^{2}\right]}, \quad \sigma_{*}=\max _{i j}\left\|X_{i j}\right\|_{\infty} .
$$

We apply the above theorem to the matrix $A-\mathbb{E}[A]$. It is easy to see that the variance of any row $\tilde{\sigma}$ is upper bounded by

$$
\tilde{\sigma} \leq \sqrt{p(1-p) n / k+q(1-q) n} \leq \sqrt{p n / k+q n},
$$

and $\sigma_{*} \leq 1$. Applying theorem A.8 with the above parameters $\tilde{\sigma}=\sqrt{p n / k+q n}$ and $\sigma_{*}=1$, we get that with probability $1-n^{-\Omega(1)}$

$$
|A-\mathbb{E}[A]| \leq 3 \sqrt{p n / k+q n}+c^{\prime} \sqrt{\log (n)} .
$$


where $c^{\prime}$ is a universal constant defined as $c^{\prime}=2 \tilde{c_{\epsilon}}$ for $\epsilon=\frac{3}{2 \sqrt{2}}-1$ and $\tilde{c_{\epsilon}}$ defined by the statement of Theorem A.8. Also note that $\mathbb{E}[A]+p I$ has the space $\mathbb{R}_{n \mid k}$ as an eigenspace with eigenvalue 0 . Therefore we have that for any unit vector $x \in \mathbb{R}_{n \mid k}$

$$
\begin{aligned}
\left|x^{T} A x\right| & \leq|A-\mathbb{E}[A]|+\left|x^{T} \mathbb{E}[A] x\right| \\
& \leq 3 \sqrt{p n / k+q n}+c^{\prime} \sqrt{\log (n)}+p \\
& \leq 3 \sqrt{p n / k+q n}+c \sqrt{\log (n)} .
\end{aligned}
$$

where $c=c^{\prime}+1$. This proves Lemma A.6

\section{A.6.2 Proof of Lemma A.7}

Proof. We prove Lemma A.7 using the following, which we prove in subsection A.6.3.

Lemma A.9. For every vertex $i$ we have that

$$
\mathbb{E}\left[\delta_{\max }^{\text {out }}(i)\right] \leq \frac{q n}{k}+28\left(\sqrt{\frac{n \log (k)}{k}} q+\log (k)\right) .
$$

Using this, the proof of lemma A.7 is as follows. Note that by a direct application of the Chernoff bound described in Corollary A.15 and with a union bound over all clusters and vertices we get that with probability $1-\frac{1}{n}$ for all vertices $i$ and all clusters $P_{t} \neq P(i)$

$$
\delta_{i \rightarrow P_{t}} \leq \frac{q n}{k}+12 \sqrt{\frac{q n}{k} \log (n)}+12 \log (n) .
$$

Lets call the above event $\mathcal{E}$ and consider the sum

$$
S(i)=\frac{\sum_{i^{\prime} \in P(i)} \delta_{\max }^{\text {out }}(i)}{n / k}
$$

Let

$$
\gamma=\frac{q n}{k}+30\left(\sqrt{\frac{n \log (k)}{k}} q+\log (k)+\sqrt{\frac{n}{k} \log (n)} \cdot \max \left\{q, \sqrt{\frac{q \log (n)}{n / k}}, \frac{\log (n)}{n / k}\right\}\right) .
$$

We have that

$$
\begin{aligned}
\mathbb{P}(\exists i S(i) \geq \gamma) & =\mathbb{P}(\mathcal{E}) \mathbb{P}(\exists i S(i) \geq \gamma \mid \mathcal{E})+\mathbb{P}(\sim \mathcal{E}) \mathbb{P}(\exists i S(i) \geq \gamma \mid \sim \mathcal{E}) \\
& \leq n^{-\Omega(1)}+\mathbb{P}(\exists i S(i) \geq \gamma \mid \sim \mathcal{E})
\end{aligned}
$$

Now for a fixed $i$ we will consider $\mathbb{P}(S(i) \geq \gamma \mid \sim \mathcal{E})$. Note that under the conditioning the individual entries in the sum above are still independent, and therefore the above is an average of independent random variables each of which is bounded by $\frac{q n}{k}+12 \sqrt{\frac{q n}{k} \log (n)}+12 \log (n)$ (by the conditioning). Also note that for any positive random variable $X$

$$
\mathbb{E}[X \mid \sim \mathcal{E}] \leq \frac{\mathbb{E}[X]}{\mathbb{P}(\sim \mathcal{E})},
$$


and since we have that $\mathbb{P}(\sim \mathcal{E}) \geq 1-1 / n$, we get that

$$
\mathbb{E}[S(i) \mid \sim \mathcal{E}] \leq \mathbb{E}[S(i)]+\frac{\mathbb{E}[S(i)]}{n-1} .
$$

We now use Hoeffding's inequality A.16 in the conditioned probability space (and remove the conditioning terms from the probability for ease of notation) to get that

$$
\mathbb{P}(S(i) \geq \mathbb{E}[S(i)]+t) \leq \exp \left(-\frac{2 \frac{n^{2}}{k^{2}} t^{2}}{\frac{n}{k}\left(\frac{q n}{k}+12 \sqrt{\frac{q n}{k} \log (n)}+12 \log (n)\right)^{2}}\right) .
$$

Now, if we choose

$$
t=25 \sqrt{\frac{n}{k} \log (n)} \cdot \max \left\{q, \sqrt{\frac{q \log (n)}{n / k}}, \frac{\log (n)}{n / k}\right\},
$$

and apply a union bound we get that with

$$
\left.\mathbb{P}\left(\exists i S_{i} \geq \mathbb{E}\left[S_{i}\right]+t \mid \sim \mathcal{E}\right]\right) \leq n^{-\Omega(1)},
$$

and now substituting the value of $\mathbb{E}[S(i) \mid \mathcal{E}]$ from before and being extremely liberal with the the contants for $n$ large enough we have that

$\mathbb{P}\left(\exists i S(i) \geq \frac{q n}{k}+30\left(\sqrt{\frac{n \log (k)}{k}} q+\log (k)+\sqrt{\frac{n}{k} \log (n)} \cdot \max \left\{q, \sqrt{\frac{q \log (n)}{n / k}}, \frac{\log (n)}{n / k}\right\}\right)\right) \leq n^{-\Omega(1)}$.

To show the second equation note that for any pair of clusters $t_{1}, t_{2}, \delta_{P_{t_{1}} \rightarrow P_{t_{2}}}$ is a sum of $(n / k)^{2}$ independent random variables. Therefore by a Chernoff bound from the second part of Theorem A.13 and a union bound we get that with probability $1-n^{-\Omega(1)}$

$$
\min _{t_{1}, t_{2}} \frac{\delta_{P_{t_{1}} \rightarrow P_{t_{2}}}}{n / k} \geq \frac{q n}{k}-2 \sqrt{q \log (n)} .
$$

\section{A.6.3 Proof of Lemma A.9}

Proof. Consider $\delta_{\max }^{\text {out }}(i)$ for some $i$, this is defined to be the maximum of $k$ random variables $S_{i}$ with $S_{i} \sim \operatorname{Bin}(n / k, q)$ (the binomial distribution with parameters $n / k, q$ ) with variance $\frac{n}{k} \sigma^{2}$ where $\sigma^{2}=q(1-q)$. Consider $\tilde{S}_{i}=S_{i}-\mathbb{E}\left[S_{i}\right]$. Let $\gamma=\sigma \sqrt{\frac{n \log (k)}{k}}+\log (k)$. From Corollary A.15 we get that

$$
\mathbb{P}\left(\tilde{S}_{i} \geq 4(t+1) \gamma\right) \leq \frac{1}{k^{t+1}}
$$

therefore by a union bound we get that the

$$
\mathbb{P}\left(\max _{i} \tilde{S}_{i} \geq 4(t+1) \gamma\right) \leq \frac{1}{k^{t}} .
$$


Hence, we can bound the expectation by

$$
\begin{aligned}
\mathbb{E}\left[\max _{i} \tilde{S}_{i}\right] & \leq 4 \gamma+\sum_{t=1}^{\infty} 4(t+1) \gamma \mathbb{P}\left(\max _{i} \tilde{S}_{i} \geq 4 t \gamma\right) \\
& \leq 4 \gamma+\sum_{t=1}^{\infty} 4(t+1) \gamma \frac{1}{k^{t-1}} \\
& \leq 4 \gamma+4 \gamma\left(\sum_{t=1}^{\infty}(t+1) \frac{1}{2^{t-1}}\right) \\
& \leq 4 \gamma+24 \gamma \\
& \leq 28\left(\sigma \sqrt{\frac{n \log (k)}{k}}+\log (k)\right)
\end{aligned}
$$

It follows from the above that

$$
\mathbb{E}\left[\delta_{\max }^{\text {out }}(i)\right] \leq \frac{n}{k} q+28\left(\sqrt{\frac{n \log (k)}{k}} q+\log (k)\right) .
$$

\section{A.6.4 Proof of Uniqueness of the solution}

In this section we prove that $Y^{*}$ is the unique optimal solution to the SDP considered in section 3. To remind the reader $M^{*}$ was the candidate dual solution. For the rest of the section we use the same notations we defined in Sction 3. To show uniqueness we make use of complementary slackness which implies that for any other optimal solution $\hat{Y}$ since with high probability $M^{*}=$ $D^{*}-\sum_{i} x_{i}^{*} R_{i}-A(G)-Z^{*}$ is an optimal solution of the dual program we have that

$$
\hat{Y} \bullet M^{*}=0 \text {. }
$$

But it is easy to see from the proof of Lemma 3.2 that we can make a stronger statement that the subspace $\mathbb{R}_{k}$ is the null space of $M^{*}$ and on the perpendicular subspace $\mathbb{R}_{n \mid k}$ the lowest eigenvalue is strictly greater than 0 . Combining this with the complementary slackness condition in particular implies that the span of the columns of $\hat{Y}$ are restricted to the span of $\mathbb{R}_{k}$. Hence, the conditions of the SDP (sum constraint, the diagonal value constraint and the positivity constraint) force $\hat{Y}=Y^{*}$ if the column space of $\hat{Y}$ is the span of $\mathbb{R}_{k}$ which proves uniqueness.

\section{A.7 Analysis for SDP in (2)}

\section{A.7.1 Proof of Theorem 2.1}

We extend the definitions of section 1.2 to ease readability. We define the notion of relative degree $\bar{\delta}$ by defining it as the number of edges present minus the number of edges not present. In this light we define the following quantities extending the definitions from Section 1.2 
$\delta_{i \rightarrow P_{t}}$ to be the "degree" of vertex $i$ to cluster $t$. Formally

$$
\begin{aligned}
\bar{\delta}_{i \rightarrow P_{t}} & \triangleq 2 \delta_{i \rightarrow P_{t}}-\left|P_{t}\right| \\
\bar{\delta}_{P_{t_{1}} \rightarrow P_{t_{2}}} & \triangleq 2 \delta_{P_{t_{1}} \rightarrow P_{t_{2}}}-\left|P_{t_{1}}\right|\left|P_{t_{2}}\right| \\
\bar{\delta}^{i n}(i) & \triangleq 2 \delta^{i n}(i)-|P(i)|
\end{aligned}
$$

We consider the following SDP in this section. Let $J$ be the $n \times n$ matrix such that $J[i, j]=1$ for all $i, j$.

$$
\begin{array}{ll}
\max & (2 * A(G)-J) \bullet Y \\
\text { s.t. } & Y_{i i}=1(\forall i) \\
& Y_{i j} \geq-\frac{1}{k-1}(\forall i, j) \\
& Y \succcurlyeq 0 .
\end{array}
$$

The dual of the above SDP is as follows

$$
\begin{array}{ll}
\min & \operatorname{Trace}(D)+\frac{1}{k-1} \sum_{i j} Z[i, j] \\
\text { s.t. } & D-Z-(2 A(G)-J) \succcurlyeq 0 .
\end{array}
$$

where $Z$ is a symmetric entrywise non-negative matrix with zeros in the diagonal and $D$ is a diagonal matrix.

The optimal solution $Y^{*}$ we have in mind is the matrix $Y_{i j}^{*}=1$ if $i, j$ belong to the same cluster and $-\frac{1}{k-1}$ if $i, j$ belong to different clusters. Note that $Y^{*}$ is PSD and is a valid solution of the primal. In this case it is easy to see that the value of the SDP is equal to

$$
(2 * A(G)-J) \bullet Y^{*}=\sum_{i}\left(\bar{\delta}_{i n}(i)-\frac{\sum_{t: P(i) \neq P_{t}} \bar{\delta}_{i \rightarrow P_{t}}}{k-1}\right)
$$

We will exhibit a candidate dual solution $D^{*}, Z^{*}$ such that

$$
(2 * A(G)-J) \bullet Y^{*}=\operatorname{Trace}(D)+\frac{1}{k-1} \sum_{i j} Z[i, j]
$$

and with high probabiltiy $D^{*}-Z^{*}-(2 A(G)-J) \succcurlyeq 0$ if condition (3) of the theorem is satisfied. Note that this implies through weak duality that $Y^{*}$ is a solution of (2). The Uniqueness of the solution can be proved exactly in the same way as in Section A.6.4

Before we define our candidate dual solution we define the following quantity for ease of notation.

$\bar{\delta}_{\text {min }} \triangleq \min _{i, j}\left(-\bar{\delta}_{i \rightarrow P(j)}-\bar{\delta}_{j \rightarrow P(i)}+\frac{\bar{\delta}_{P(j) \rightarrow P(i)}}{(n / k)}\right)=\left(n / k-2 \max _{i, j}\left(\delta_{i \rightarrow P(j)}+\delta_{j \rightarrow P(i)}-\frac{\delta_{P(j) \rightarrow P(i)}}{(n / k)}\right)\right)$

We begin by describing the choice of $Z^{*}$. If vertex $i$ and $j$ belong to the same clusters then $Z^{*}[i, j]=0$ otherwise

$Z^{*}[i, j] \triangleq\left(-\frac{\bar{\delta}_{i \rightarrow P(j)}}{n / k}-\frac{\bar{\delta}_{j \rightarrow P(i)}}{n / k}+\frac{\bar{\delta}_{P(j) \rightarrow P(i)}}{(n / k)(n / k)}-\frac{\bar{\delta}_{\text {min }}}{n / k}\right)=\left(1-2\left(\frac{\delta_{i \rightarrow P(j)}}{n / k}+\frac{\delta_{j \rightarrow P(i)}}{n / k}-\frac{\delta_{P(j) \rightarrow P(i)}}{(n / k)(n / k)}\right)-\frac{\bar{\delta}_{\text {min }}}{n / k}\right)$ 
Note that by definition (23) $Z^{*}$ is a symmetric non-negative matrix. We now define the diagonal matrix $D^{*}$ as

$$
D^{*}[i, i] \triangleq \delta_{i n}^{-}(i)+\bar{\delta}_{\text {min }}=2\left(\delta_{i n}(i)-\max _{i, j}\left(\delta_{i \rightarrow P(j)}+\delta_{j \rightarrow P(i)}-\frac{\delta_{P(j) \rightarrow P(i)}}{(n / k)}\right)\right)
$$

A simple calculation now shows the first required property that

$$
\operatorname{Trace}(D)+\frac{1}{k-1} \sum_{i j} Z[i, j]=\sum_{i}\left(\bar{\delta}_{i n}(i)-\frac{\sum_{t: P(i) \neq P_{t}} \bar{\delta}_{i \rightarrow P_{t}}}{k-1}\right)=(2 * A(G)-J) \bullet Y^{*}
$$

We now proceed to show that $D^{*}, Z^{*}$ is a valid dual solution, i.e.

$$
M^{*}=D^{*}-Z^{*}-(2 A-J) \succeq 0
$$

To see this consider the following extension of the decomposition of the space $\mathbb{R}^{n}$ defined in section 3 .

Definition A.10. Given a $k$-clustering of $n$ vertices $\left\{P_{t}\right\}_{t=1}^{k}$ we define the vectors $v_{t}$ to be the indicator vectors of the clusters. We further define the following subspaces, which are perpendicular to each other, and partition $\mathbb{R}^{n}$.

- $\mathbb{1}$ : the vectors with 1 in each coordinate

- $\mathbb{R}_{k-1}$ : the $k-1$ dimensional subspace such that for every vector $v \in \mathbb{R}_{k-1}, v(i)=v(j)$ if $P(i)=P(j)$ and $<v, \mathbb{1}\rangle=0$

- $\mathbb{R}_{n \mid k}$ : the subspace perpendicular to $\mathbb{R}_{k-1} \cup \mathbb{1}$, i.e. the subspace where the sum on each cluster is equal to 0 .

Following are two easy observations that follow from simple calculations similar to the calculations shown in Section 3.

Observation A.11. $\left(\forall v \in \mathbb{R}_{k-1}\right)\left(D^{*}-Z^{*}-(2 A-J)\right) v=0$

Observation A.12. $\left(\forall v \in \mathbb{R}_{n \mid k}\right) v^{T} Z^{*} v=0$

We first focus on the subspace $\mathbb{R}_{n \mid k}$ and show that $\forall x \in \mathbb{R}_{n \mid k}$

$$
x^{T}\left(D^{*}-Z^{*}-(2 A-J) x=x^{T}\left(D^{*}-2 A\right) x \geq 0\right.
$$

The proof of the above statement follows from the following set of inequalities

$$
\begin{aligned}
x^{T}\left(D^{*}-2 A\right) x \geq \min _{i} & D^{*}[i, i]-2 \max _{x} x^{T} A(G) x \\
& \geq 2 \min _{i} \nu(i)-2 \max _{x} x^{T} A(G) x \\
& \geq 2\left(\min _{i} \nu(i)-\hat{c}(\sqrt{p n / k+q n}+\sqrt{\log (n)})\right) \\
& \geq 0
\end{aligned}
$$

where the second inequality above follows from substituting the values of $\bar{\delta}_{i \rightarrow P(t)}$ in terms of $\delta_{i \rightarrow P(t)}$ in the expression for $D^{*}[i, i]$ and using the definition of $\nu(i)$. The second inequality follows from 
Lemma A.6 and third inequality follows from the condition (3). Note that in condition (3) if we assume the constant to be $\hat{c}+1$ instead of $\hat{c}$ then we get a stronger property that the above quantity is in fact greater than $\sqrt{\log (n)}$ and not just positive. We use this below.

The above analysis shows that the matrix $M^{*}=D^{*}-Z^{*}-(2 A-J)$ is PSD on the subspace $\mathbb{R}_{n \mid k}$. Lets now focus on a vector $y \in \mathbb{R}_{n \mid k} \oplus \mathbb{1}$. Let $H^{*}=D^{*}-Z^{*}-2 A=M^{*}-J$. By appropriate scaling we can consider any $y=x+\delta \frac{\mathbb{1}}{\sqrt{n}}$ (see footnote ${ }^{1}$ ) where $x \in \mathbb{R}_{n \mid k}$ is a unit vector and $\delta \geq 0$. In the analysis above we explained that $x^{T} H^{*} x \geq \sqrt{\log (n)}\|x\|^{2}=\sqrt{\log (n)}$. With these facts in place consider $y^{T} M^{*} y$

$$
\begin{aligned}
y^{T} M^{*} y & =x^{T} H^{*} x+\frac{\delta^{2}}{n} \mathbb{1}^{T} J \mathbb{1}+2 x^{T} H^{*} \frac{\delta}{\sqrt{n}} \mathbb{1} \\
& \geq \sqrt{\log (n)}+\delta^{2} n-2 \delta\left\|H^{*}\right\|
\end{aligned}
$$

where we use the fact that for unit vector $x \frac{x^{T} H^{*} \mathbb{1}}{\sqrt{n}} \leq\left\|H^{*}\right\|$. Therefore as long as we have that $4\left\|H^{*}\right\|^{2} \leq 4 n \sqrt{\log (n)}$ we have that that $y^{T} M^{*} y \geq 0$ (as the expression is a quadratic in $\delta$ ). Therefore we need to control the spectral norm of $H^{*}$. We can show the above via very simple and fairly loose calculations

$$
\begin{aligned}
\left\|H^{*}\right\| & \leq\left\|D^{*}\right\|+2\|A\|+\left\|Z^{*}\right\| \\
& \leq \max D^{*}[i, i]+2 \delta_{\max }+O\left(\delta_{\max }\right) \\
& \leq O\left(\delta_{\max }\right)
\end{aligned}
$$

where $\delta_{\max }$ is the degree of the vertex with maximum degree in the graph $G$. The above equation follows with very loose aproximations from the definitions. A simple chernoff bound shows that with high probability $\delta_{\max } \leq p m+k q m+\sqrt{p m+k q m} \log (n) \leq O\left(k \log (n)+\log ^{3 / 2}(n)\right)$ where we have replaced $p$ with $\alpha \frac{\log (m)}{m}$ and $q$ with $\beta \frac{\log (m)}{m}$ which implies that $\left\|H^{*}\right\| \leq \sqrt{n}$ which completes the proof since we have shown that $M^{*}$ is PSD.

\section{A.8 Monotone Adversary - Proof of Theorem 4.1}

Proof. We consider the SDP relaxation (4) as in the proof of Theorem 1.1. Let $Y^{*}(G)$ be the optimal solution of the SDP when we run it on the graph $G$. Now suppose $G \sim \mathcal{G}_{p, q, k}$. The proof of Theorem 1.1 shows that with high probability, $Y^{*}(G)$ is unique and it corresponds to the hidden partition. Suppose this event happens, we then show that for any graph $G_{a d v}$ generated by the monotone adversary after acting on $G, Y^{*}\left(G_{a d v}\right)$ is also unique and it is equal to $Y^{*}(G)$. This will prove Theorem 4.1.

Define $S D P_{G}(Y)$ to be the objective value (corresponding to the graph $G$ ) of a feasible matrix $Y$, i.e. $S D P_{G}(Y)=A(G) \bullet Y$. Note that since $Y$ has only positive entries (since it is a feasible solution) we have that $A\left(G^{\prime}\right) \bullet Y \leq A(G) \bullet Y$, if $G^{\prime}$ is a subgraph of $G$. Also since $Y \succeq 0$ and its diagonal entries $Y_{i i}=1$ we have that $\left|Y_{i j}\right| \leq 1$. Therefore $A(G \cup e) \bullet Y \leq A(G) \bullet Y+2$. Suppose the monotone adversary adds a total of $r^{+}$edges and removes $r^{-}$edges. From the monotonicity

\footnotetext{
${ }^{1}$ Indeed by definition any vector $y \in \mathbb{R}_{n \mid k} \oplus \mathbb{1}$ can be written as $x+\delta \frac{\mathbb{1}}{\sqrt{n}}$ for some $\delta$ and $x \in \mathbb{R}_{n \mid k}$. For the purpose of proving positive definiteness we can always divide by any positive number and can there fore consider $\frac{y}{\|x\|}$. Also note that we can consider $y$ or $-y$ equivalently and hence can consider the case when $\delta>0$.
} 
of the adversary it is easy to see that $A\left(G_{a d v}\right) \bullet Y^{*}(G)=A(G) \bullet Y^{*}(G)+2 r^{+}$. However for any other solution by the argument above we have that $A\left(G_{a d v}\right) \bullet Y \leq A(G) \bullet Y+2 r^{+}$. Also by our assumption we have that $A(G) \bullet Y^{*}(G)<A(G) \bullet Y$ for any feasible $Y \neq Y^{*}(G)$. Putting it together we have that

$$
A\left(G_{a d v}\right) \bullet Y^{*}(G)=A(G) \bullet Y^{*}(G)+2 r^{+}>A(G) \bullet Y+2 r^{+} \geq A\left(G_{a d v}\right) \bullet Y,
$$

for any feasible $Y \neq Y^{*}(G)$, which proves the theorem.

\section{A.9 Forms of Chernoff Bounds and Hoeffding Bounds Used in the Arguments}

Theorem A.13 (Chernoff). Suppose $X_{1} \ldots X_{n}$ be independent random variables taking values in $\{0,1\}$. Let $X$ denote their sum and let $\mu=\mathbb{E}[X]$ be its expectation. Then for any $\delta>0$ it holds that

$$
\begin{aligned}
& \mathbb{P}(X>(1+\delta) \mu)<\left(\frac{e^{\delta}}{(1+\delta)^{(1+\delta)}}\right)^{\mu}, \\
& \mathbb{P}(X<(1-\delta) \mu)<\left(\frac{e^{-\delta}}{(1-\delta)^{(1-\delta)}}\right)^{\mu} .
\end{aligned}
$$

A simplified form of the above bound is the following formula (for $\delta \leq 1$ )

$$
\begin{aligned}
& \mathbb{P}(X \geq(1+\delta) \mu) \leq e^{-\frac{\delta^{2} \mu}{3}}, \\
& \mathbb{P}(X \leq(1-\delta) \mu) \leq e^{-\frac{\delta^{2} \mu}{2}} .
\end{aligned}
$$

Theorem A.14 (Bernstein). Suppose $X_{1} \ldots X_{n}$ be independent random variables taking values in $[-M, M]$. Let $X$ denote their sum and let $\mu=\mathbb{E}[X]$ be its expectation, then

$$
\mathbb{P}(|X-\mu| \geq t) \leq \exp \left(-\frac{1}{2} \frac{t^{2}}{\sum_{i} \mathbb{E}\left[\left(X_{i}-\mathbb{E}\left[X_{i}\right]\right)^{2}\right]+M t / 3}\right) .
$$

Corollary A.15. Suppose $X_{1} \ldots X_{n}$ are i.i.d Bernoulli variables with parameter $p$. Let $\sigma=$ $\sigma\left(X_{i}\right)=p(1-p)$ then we have that for any $r \geq 0$

$$
\mathbb{P}(X \geq \mu+\alpha \sigma \sqrt{n \log (r)}+\alpha \log (r)) \leq e^{-\frac{\alpha \log (r)}{4}} .
$$

Proof. We have that $n \sigma^{2}=n p(1-p)$ and $M=1$. We can now choose $t=\alpha \sigma \sqrt{n \log (r)}+\alpha \log (r)$. This implies that $\frac{n \sigma^{2}+t / 3}{t^{2}} \leq \frac{1}{\log (r)}\left(1 / \alpha^{2}+1 / 3 \alpha\right) \leq \frac{2}{\alpha \log (r)}$ which implies from Theorem A.14 that $\mathbb{P}(X>\mu+\alpha \sigma \sqrt{n \log (r)}+\alpha \log (r)) \leq e^{-\frac{\alpha \log (r)}{4}}$.

Theorem A.16 (Hoeffding). Let $X_{1} \ldots X_{n}$ be independent random variables. Assume that the $X_{i}$ are bounded in the interval $\left[a_{i}, b_{i}\right]$. Define the empirical mean of these variables as

$$
\bar{X}=\frac{\sum_{i} \bar{X}_{i}}{n}
$$

then

$$
\mathbb{P}(|\bar{X}-\mathbb{E}[\bar{X}]| \geq t) \leq 2 \exp \left(-\frac{2 n^{2} t^{2}}{\sum_{i=1}^{n}\left(b_{i}-a_{i}\right)^{2}}\right)
$$

